\title{
Bulk locality and cooperative flows
}

\author{
Veronika E. Hubeny \\ Center for Quantum Mathematics and Physics (QMAP), \\ Department of Physics, University of California, \\ Davis, CA 95616 U.S.A. \\ E-mail: veronika@physics.ucdavis.edu
}

ABstract: We use the 'bit thread' formulation of holographic entanglement entropy to highlight the distinction between the universally-valid strong subadditivity and the more restrictive relation called monogamy of mutual information (MMI), known to hold for geometrical states (i.e. states of holographic theories with gravitational duals describing a classical bulk geometry). In particular, we provide a novel proof of MMI, using bit threads directly. To this end, we present an explicit geometrical construction of cooperative flows which we build out of disjoint thread bundles. We conjecture that our method applies in a wide class of configurations, including ones with non-trivial topology, causal structure, and time dependence. The explicit nature of the construction reveals that MMI is more deeply rooted in bulk locality than is the case for strong subadditivity.

KEYwords: AdS-CFT Correspondence, Gauge-gravity correspondence

ArXIV EPRINT: 1808.05313 


\section{Contents}

1 Introduction 1

2 Bit thread proof of MMI - overview 5

$\begin{array}{lll}3 & \text { Basics of cooperative flow construction } & 7\end{array}$

$\begin{array}{lll}3.1 \text { Setup } & 7\end{array}$

3.2 Cooperative flows for uncorrelated regions 9

$\begin{array}{ll}3.3 \text { Motivation for our strategy } & 10\end{array}$

$\begin{array}{ll}3.4 \text { Flow construction } & 12\end{array}$

$\begin{array}{lll}3.4 .1 & \text { Maximizer flow partitions } & 13\end{array}$

$\begin{array}{lll}3.4 .2 & \text { Foliation building blocks } & 14\end{array}$

$\begin{array}{lll}\text { 3.4.3 Foliation for each thread bundle, smooth flows } & 16\end{array}$

3.4.4 Single local foliation for all thread bundles, patched flows $\quad 18$

4 Generalizations $\quad 20$

$\begin{array}{lll}4.1 & \text { Multiple sub-partitions } & 21\end{array}$

4.2 Exciting the geometry 24

$\begin{array}{lll}4.3 & \text { Higher dimensions } & 27\end{array}$

$\begin{array}{ll}4.4 \text { Time dependence } & 27\end{array}$

$\begin{array}{lll}5 & \text { Discussion } & 29\end{array}$

A 5-party cyclic inequality 33

\section{Introduction}

Entanglement, a quintessentially quantum characteristic, is expected to play a key role in holography, not only as a powerful tool in describing a given physical system, but also as a fundamental building block in the duality itself. Understanding precisely how this works has been one of the outstanding goals in the holography program over the last few years. The basic quantity of interest is entanglement entropy, which provides a particularly natural measure of entanglement, and from which other useful quantities are built. Universal relations between these quantities allow us to gain further insights, and in the holographic context may even diagnose whether a given quantum field theory state has a holographic dual corresponding to a classical geometry. The way in which the bulk geometry implements these relations can then provide crucial insights to unraveling the emergence of spacetime in holography.

In the following, we will focus on one particular relation known as monogamy of mutual information (MMI). In order to explain its significance, we first review its construction in 
the broader context of other relations. ${ }^{1}$ The entanglement entropy (EE) of a system $A$ can be thought of as a measure of mixedness of its reduced density matrix $\rho_{A}$ obtained by tracing out the complement $\bar{A}$ in a specified bi-partitioning of the system $\mathcal{H}=\mathcal{H}_{A} \otimes \mathcal{H}_{\bar{A}}$. Explicitly, EE is defined as the von Neumann entropy

$$
S(A)=-\operatorname{Tr} \rho_{A} \log \rho_{A} .
$$

Refining the partition into several subsystems $A, B$, etc., we can consider general constraints on the respective entanglement entropies. ${ }^{2}$ For example, among particularly useful relations are sub-additivity (SA), strong sub-additivity (SSA) and its cousin (related by purification) weak monotonicity (WM), and the above-mentioned monogamy of mutual information (MMI), which can respectively be expressed as:

$$
\begin{array}{rlrl}
(\mathrm{SA}) & : & S(A)+S(B) & \geq S(A B) \\
(\mathrm{SSA}): & & S(A B)+S(B C) & \geq S(B)+S(A B C) \\
(\mathrm{WM}): & S(A B)+S(B C) & \geq S(A)+S(C) \\
(\mathrm{MMI}): & & S(A B)+S(B C)+S(C A) & \geq S(A)+S(B)+S(C)+S(A B C)
\end{array}
$$

where we use the shorthand $A B \equiv A \cup B$, etc. While these relations look superficially similar, there is an important difference between the first three and the last one: SA, SSA, and WM are all valid for any quantum system in any state and for any partition. MMI, on the other hand, is a stronger relation which does NOT hold in general, but as initially proved in [3], it does hold for holographic states.

To digest the significance of this statement, it is useful to rewrite the above relations in a slightly more suggestive way. One can think of the triangle inequality type relation of SA (1.2) as the statement of positivity of the mutual information

$$
I(A: B) \equiv S(A)+S(B)-S(A B),
$$

which characterizes the amount of correlations (both classical and quantum) between the systems $A$ and $B$. One can similarly view the convexity property of SSA (1.3) as the statement of positivity of the conditional mutual information

$$
I(A: C \mid B) \equiv I(A: B C)-I(A: B),
$$

which says that the amount of correlation is monotonic under inclusion. These two statements, that the amount of correlations cannot be negative and that enlarging the system cannot decrease the amount of correlations with another system, are intuitively clear. ${ }^{3}$

\footnotetext{
${ }^{1}$ In the interest of being reasonably self-contained, in the next few paragraphs we give a very brief review of these relations. This is well-known material; for a nice review of the relations and their proofs in the holographic context (for time reflection symmetric configurations), see e.g. [1].

${ }^{2}$ It is known that even in the restricted context of holography [2], there are in fact infinitely many such relations if we allow arbitrary number of partitions, though the complete set has hitherto remained elusive.

${ }^{3}$ Though perhaps surprisingly so; see [4] for an exposition of some of the subtleties.
} 
The statement of MMI (1.5), on the other hand, translates to the statement of negativity of the tripartite information ${ }^{4}$

$$
I_{3}(A: B: C) \equiv I(A: B)+I(A: C)-I(A: B C),
$$

which can therefore be thought of as measuring the non-extensivity of mutual information. If the correlations between the three systems are purely quantum, then they must obey the property known as monogamy of entanglement (implying that quantum entanglement between $A$ and $B$ cannot be shared by $C$ etc.), so the entanglement contributing to $I(A: B)$ cannot comprise any part of contribution to $I(A: C)$ and vice-versa. That in turn means that $I(A: B C)$ must be at least as large as the individual contributions, $I(A: B)+I(A: C)$, implying the MMI relation (1.5) (and motivating its name). If, on the other hand, the correlations are classical, then they can be redundantly shared, allowing for the possibility of positive tripartite information. ${ }^{5}$ In the holographic context, then, the fact that MMI holds (i.e. that $I_{3} \leq 0$ ) is rather suggestive of sufficient quantumness of the correlations between subsystems. ${ }^{6}$ This emphasizes the important point that while we tend to think of the bulk as behaving classically, this is merely an effective description: the corresponding CFT state manifests quantum features even with respect to the observables naturally associated with the bulk variables.

One of the advantages of the bulk description is that it often conveniently geometrizes highly non-trivial statements about the system, allowing them to be proved with ease, at least in a certain context. ${ }^{7}$ The inequalities (1.2)-(1.5) provide an excellent example of this intriguing fact. According to the Ryu-Takayanagi (RT) prescription [6, 7] pertaining to static situations, the entanglement entropy of a given spatial region $A$ in the boundary $\mathrm{CFT}^{8}$ is computed by quarter of the proper area (in Planck units) of a minimal surface ${ }^{9}$ $\mathfrak{m}_{A}$ which is homologous to $A$ (and hence anchored on the entangling surface $\partial A$ ):

$$
S(A)=\frac{1}{4 G_{N}} \text { Area }\left[\mathfrak{m}_{A}\right] \text {. }
$$

In arbitrary time-dependent situations, this relation naturally generalizes to the HubenyRangamani-Takayanagi (HRT) prescription [9], wherein the minimal surface $\mathfrak{m}_{A}$ at constant

\footnotetext{
${ }^{4}$ One could argue that 'negative tripartite information' (also known as interaction information) is a more natural quantity to consider, since its positivity appears in a variety of QI contexts on similar footing as other positive quantities. However, to avoid confusion, we will presently stick to the usual convention of using the standard $I_{3}$.

${ }^{5}$ In fact even the $n \geq 4$ party GHZ state $\frac{1}{\sqrt{n}}(|00 \ldots 0\rangle+|11 \ldots 1\rangle)$, which in some regards is highly quantum but whose correlations are maximally redundant, violates MMI.

${ }^{6}$ This has already been suggested by [3] and is further supported by the recent exploration of [5] using the relative entropy of entanglement as a better measure of quantumness of correlations.

${ }^{7}$ In everything that follows, we will focus on the leading order in the large- $N$, large- $\lambda$ limit of the CFT, which corresponds to a classical geometry in the bulk. (However we briefly comment on quantum and stringy correction in section 5.)

${ }^{8}$ Although in our gauge theory context, the Hilbert space factorization for such a spatial partitioning strictly-speaking does not apply, this does not affect any of the results discussed below, as can be justified using the more rigorous framework of Tomita-Takesaki theory based on algebra of observables; for a recent review see e.g. [8].

${ }^{9}$ The surface is by definition of co-dimension-two in the full bulk spacetime, which in the static context can be more usefully viewed as spatially co-dimension-one within a constant time slice.
} 
time gets simply replaced by a spacetime co-dimension-two extremal surface $\mathfrak{E}_{A}$ homologous to $A$ (and in case of multiple such candidates, being the one with the smallest area). However, in what follows we will primarily focus on the static case.

Remarkably, the proof of SSA in the static case, first obtained in [10] and later generalized in [3], follows almost immediately from (1.9). As we briefly review in section 3.1 in a simple setup, one can obtain (1.3) by comparing the respective bulk minimal surface areas and using the fact that a minimal surface by definition cannot have larger area than any other surface in the same homology class. Interestingly, the argument for MMI is virtually identical [3] — indeed, as indicated below, in this context the proof of MMI actually reduces to that of SSA, despite the fact that these are logically distinct relations with different physical meanings. This curious statement likewise holds in the general time-dependent setting [11]. In other words, the RT and HRT prescriptions do not discern the fundamental distinction between SSA and MMI. Hence their virtue, that the bulk repackaging simplifies the dual CFT description, turns into somewhat of a detriment, by obscuring a crucial aspect of these relations. ${ }^{10}$

To elucidate this important distinction, then, we need to go beyond the RT (or HRT) prescription. Of course the structural difference between SSA and MMI would become more apparent if we include quantum corrections, but going beyond the classical limit does not address the essence of the puzzle directly. Fortuitously, however, we in fact can see a distinction already at the leading order in large- $N$ description, as explained below. This is manifested by utilizing a different geometrical prescription for holographic entanglement entropy, namely the "bit thread" formulation of Freedman and Headrick [12]. This prescription, reviewed in section 2, captures the quantum information theoretic meaning of the relevant constructs more naturally. ${ }^{11}$ Here entanglement entropy is given by the maximum flux of a bulk 'flow' (defined as a divergence-free vector field of bounded norm), whose integral curves are the bit threads. As shown in [12], the statements of SA and SSA can be recast into statements of positivity of the number of threads obeying certain restrictions - and since this number manifestly cannot be negative, these two relations are then even more directly evident in the bit thread formulation than via RT.

However, as already emphasized in [12], the MMI relation does not lend itself to such an easy argument; in fact, [12] has not been able to find a proof directly in the flow language without recourse to the equivalence with RT. The bit thread formulation, then, provides a useful window of opportunity: a framework wherein the distinction between SSA and MMI should become more directly apparent, which we can use to elucidate the role of the geometrization and ultimately the emergence of bulk spacetime. More specifically, [12] demonstrates that just using the basic building blocks of the flows (and its crucial properties such as 'nesting') does not guarantee MMI: some other non-trivial property of flows is needed. In this paper, we set out to elucidate this property. We will find that it is deeply rooted in bulk locality.

\footnotetext{
${ }^{10}$ There are several other features, such as the bulk location of the CFT information, for which these prescriptions likewise seem more puzzling than suggestive.

${ }^{11}$ Although, as argued in [12] using the Max Flow-Min Cut theorem and shown more explicitly in [13], the bit thread prescription for holographic entanglement entropy is mathematically equivalent to the RT prescription, it nevertheless turns out to be much better tailored to the present considerations.
} 
In particular, we prove MMI directly using the language of bit threads. ${ }^{12}$ The structure of the presentation is as follows. Our argument starts with the observation ${ }^{13}$ (explained in section 2), that under the assumption of the existence of certain 'cooperative flows', MMI would follow easily. The crux of the argument then is to demonstrate the existence of such cooperative flows. We achieve this by an explicitly constructive method. In section 3 we explain the basic idea in a simple context, and in section 4 we generalize this construction to arbitrary spatial partitions of CFT states describing arbitrary asymptotically AdS geometries. We then revisit the relation to bulk locality and discuss further implications and open questions in section 5 .

\section{Bit thread proof of MMI - overview}

To approximate a partitioning of the Hilbert space $\mathcal{H}=\mathcal{H}_{A} \otimes \mathcal{H}_{B} \otimes \mathcal{H}_{C} \otimes \mathcal{H}_{D}$ (with $D \equiv \overline{A B C}$ being the purifier) in the holographic context, we typically let $A, B, C$, and $D$ correspond to spatial regions partitioning the space on which the theory lives. ${ }^{14}$ Recall that the general form of MMI, written in a manifestly $A B C$-cyclically symmetric form, is

$$
S(A B)+S(B C)+S(C A) \geq S(A)+S(B)+S(C)+S(A B C),
$$

where each term gives the entanglement entropy of the corresponding boundary subregion.

In this section, we outline the basic argument for demonstrating (2.1) in the holographic context using the bit thread formulation. Following [12], we can describe the entanglement entropy in terms of bulk flows. A flow $v$ is defined as divergenceless vector field of bounded norm,

$$
\nabla \cdot v=0, \quad|v| \leq 1
$$

whose integral curves are dubbed 'bit threads'. The entanglement entropy of a given spatial region $X$ on the boundary is computed by maximizing the flux through $X$ over all bulk flows,

$$
S(X)=\max _{v} \int_{X} v
$$

where we're using the shorthand $\int_{X} v:=\int_{X} \sqrt{h} n_{\mu} v^{\mu}=\int_{\mathfrak{m}_{X}} \sqrt{h} n_{\mu} v^{\mu}$ (with $n_{\mu}$ being the unit normal, and $h$ the determinant of the induced metric, on the integration surface which by homology and Stokes' theorem can be taken at $\mathfrak{m}_{X}$ ). Any flow which maximizes the flux

\footnotetext{
${ }^{12}$ The authors of [14] (whom we thank for sharing an advance version of their draft) likewise have a proof of MMI, inspired by previously known theorems regarding multicommodity flows, using tools of the theory of convex optimization (in particular the Lagrangian duality) along similar lines as [13], to abstractly argue for the existence of 'cooperative flows'. Our argument is complementary to that line of reasoning: it is explicitly constructive and directly geometrical.

${ }^{13}$ We thank Matt Headrick for sharing this observation, which inspired the present work (as well as the term 'cooperative flows').

${ }^{14}$ For the CFT living on a single connected spacetime, this restriction corresponds to taking a pure state, so the bulk admits no eternal black hole horizons. However, it is easy to generalize to the case with black holes (or even more generally 'multi-boundary wormholes') by letting $D$ (as well as $A, B$, and $C$ ) include regions in multiple disconnected boundary spacetimes.
} 
through a region $X$ is called a maximizer flow for the region $X$ and denoted by ${ }^{15} v_{X}$, so the entanglement entropy can be characterized more simply as the flux of a maximizer flow,

$$
S(X)=\int_{X} v_{X} \geq \int_{X} v
$$

where the inequality by definition holds for any flow $v$. Note that a maximizer flow $v_{X}$ for a given region $X$ is highly non-unique: the restriction (2.2) only fully fixes it at the minimal surface $\mathfrak{m}_{X}$ (through which it must pass perpendicularly and with unit norm). For later purposes it is also useful to note that switching the direction of given a maximizer flow $v_{X}$ for region $X$ automatically generates a maximizer flow $v_{\bar{X}}=-v_{X}$ for its complement $\bar{X}$.

Applying (2.4) to the l.h.s. of the MMI inequality (2.1), we have

$$
\begin{aligned}
S(A B)+S(B C)+S(C A) & =\int_{A B} v_{A B}+\int_{B C} v_{B C}+\int_{C A} v_{C A} \\
& \geq \int_{A B} v_{1}+\int_{B C} v_{2}+\int_{C A} v_{3} \\
& =\int_{A}\left(v_{1}+v_{3}\right)+\int_{B}\left(v_{1}+v_{2}\right)+\int_{C}\left(v_{2}+v_{3}\right),
\end{aligned}
$$

where the inequality holds for arbitrary flows $v_{1}, v_{2}$, and $v_{3}$. To relate this to the r.h.s. of $\left(2.1\right.$ ), we want to choose these flows $v_{i}$ (with $i=1,2,3$ ) to be given by

$$
\begin{aligned}
& v_{1}=\tilde{v}_{1} \equiv \frac{1}{2}\left(v_{A}+v_{B}-v_{C}+v_{A B C}\right) \\
& v_{2}=\tilde{v}_{2} \equiv \frac{1}{2}\left(-v_{A}+v_{B}+v_{C}+v_{A B C}\right) \\
& v_{3}=\tilde{v}_{3} \equiv \frac{1}{2}\left(v_{A}-v_{B}+v_{C}+v_{A B C}\right)
\end{aligned}
$$

where $v_{A}, v_{B}, v_{C}$, and $v_{A B C}$ are maximizer flows for the corresponding regions. It is easy to see that in such a case the r.h.s. of (2.5) would reduce to

$$
\begin{aligned}
& \int_{A}\left(\tilde{v}_{1}+\tilde{v}_{3}\right)+\int_{B}\left(\tilde{v}_{1}+\tilde{v}_{2}\right)+\int_{C}\left(\tilde{v}_{2}+\tilde{v}_{3}\right) \\
= & \left.\int_{A}\left(v_{A}+v_{A B C}\right)+\int_{B}\left(v_{B}+v_{A B C}\right)+\int_{C}\left(v_{C}+v_{A B C}\right)\right) \\
= & S(A)+S(B)+S(C)+S(A B C),
\end{aligned}
$$

thereby proving the MMI relation.

The crux of the argument then boils down to showing that the objects $\tilde{v}_{i}$ defined by (2.6) are indeed flows, i.e. that they satisfy (2.2) and hence we're allowed to equate them to the l.h.s. terms in (2.6). While divergencelessness of $\tilde{v}_{i}$ 's is guaranteed by linearity, the norm bound need not a-priori remain satisfied: ay triangle inequality we're only guaranteed

\footnotetext{
${ }^{15}$ In the interest of compactness of notation, here we deviate from [12] (which uses the notation $v(X)$ for the maximizer flow). To label multiple generic flows which are not necessarily maximizing for any region, we will use a numerical subscript, e.g. $v_{1}, v_{2}$, etc. (not to be confused with a letter subscript which labels a region and signifies maximizer flow through that region).
} 
that the average $\frac{1}{2}\left(v_{\alpha}+v_{\beta}\right)$ of any two flows $v_{\alpha}$ and $v_{\beta}$ is itself a flow, whereas the expressions involving 4 flows in (2.6) can exceed unit norm (and in fact one can easily construct maximizer flows $v_{A}, v_{B}, v_{C}$, and $v_{A B C}$, for which each of $\left|\tilde{v}_{1}\right|,\left|\tilde{v}_{2}\right|$, and $\left|\tilde{v}_{3}\right|$ becomes 2 somewhere).

However, not all maximizer flows $v_{A}, v_{B}, v_{C}$, and $v_{A B C}$ have this undesirable property. Given any specified regions $A, B$, and $C$, we can find maximizer flows $v_{A}, v_{B}, v_{C}$, and $v_{A B C}$ which guarantee that $\left|\tilde{v}_{1}\right| \leq 1,\left|\tilde{v}_{2}\right| \leq 1$, and $\left|\tilde{v}_{3}\right| \leq 1$ simultaneously throughout the bulk. Below, we outline an explicit construction (in fact two natural ones) of maximizer flows which satisfy this requirement, thus supplying the missing step in the proof of MMI. Our construction utilizes the observation [13] that a foliation of a bulk region by minimal surfaces induces a maximally-collimated flow; we demonstrate that suitable foliations always exist which 'comb' the flows $v_{A}, v_{B}, v_{C}$, and $v_{A B C}$ in such a way as to 'cooperate' in the requisite fashion.

\section{Basics of cooperative flow construction}

We start with a simple class of examples to illustrate the basic idea, and subsequently generalize our arguments to more complicated situations in section 4. In particular, we first fix convenient dimensionality and state, but consider a generic partition within a given topology class.

\subsection{Setup}

Let us consider pure $\mathrm{AdS}_{3}$ geometry, with all regions of interest localized at a fixed time $t=0$, so that we can WLOG restrict attention to bulk spatial slice, i.e. the Poincare disk. ${ }^{16}$ We will consider the boundary to live on $S^{1}$ space and take the partitions $A, B$, and $C$ to be simple adjoining intervals. We will denote the complementary region $\overline{A B C} \equiv D$ and assume the total state to be pure, i.e. $S(A B C D)=0$. A representative of a generic such configuration is sketched in figure 1 , along with the corresponding minimal surfaces relevant for the terms appearing in MMI (2.1). Note that $S(A B C)=\frac{1}{4}$ Area $\left[\mathfrak{m}_{D}\right]$ and

$$
S(A C)=\frac{1}{4} \min \left\{\text { Area }\left[\mathfrak{m}_{A}\right]+\text { Area }\left[\mathfrak{m}_{C}\right], \text { Area }\left[\mathfrak{m}_{B}\right]+\text { Area }\left[\mathfrak{m}_{D}\right]\right\} .
$$

We can now readily confirm that the RT-based geometric proof of MMI is then manifestly identical $^{17}$ to the SSA proof of [10]: for either option in the r.h.s. of $(3.1), S(A C)$ is canceled by two of the terms on the r.h.s. of (2.1), with the remaining terms equivalent to either SSA (1.3), or its purification WM (1.4). In the present configuration these two relations follow from cutting and rejoining $\mathfrak{m}_{A B}$ and $\mathfrak{m}_{B C}$ so as to implement $A C$ homology, and observing that each surface necessarily has greater area than the globally minimal surface

\footnotetext{
${ }^{16}$ We will only use the metric details of $\mathrm{AdS}_{3}$ for generating the explicit plots, but otherwise our arguments will be robust to deforming the geometry. Moreover, as long as we're restricting to static context, our results will not depend on assuming any specific field equations, energy conditions, etc.

${ }^{17}$ Note that our present argument based on cancelation of surfaces hinges on the fact that our "minimal surfaces' are one-dimensional; in higher dimensions one has to recourse to the more refined argument of [3].
} 


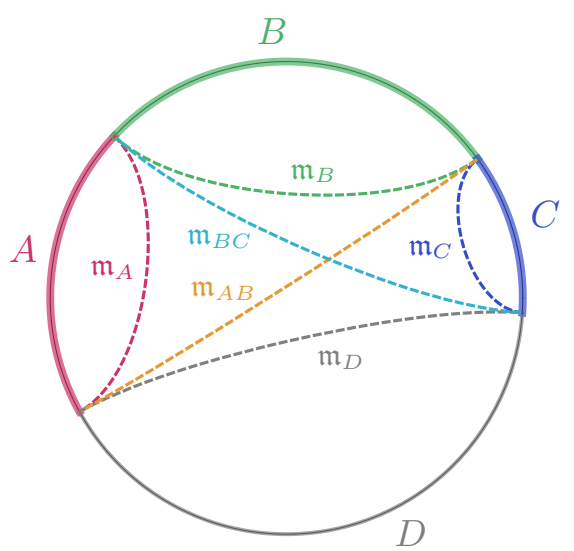

Figure 1. An example of partitioning of the boundary space into regions (thick curves) $A, B$, $C$, and the complement $\overline{A B C} \equiv D$, as labeled. The corresponding minimal surfaces (dashed curves) for these 4 regions, along with those for $A B$ and $B C$ are also indicated. (In this and subsequent plots, we plot the Poincare disk in global coordinates described by constant $t$ slice of $d s^{2}=\frac{1}{\cos ^{2} \rho}\left[-d t^{2}+d \rho^{2}+\sin ^{2} \rho d \varphi^{2}\right]$ with $\rho=\pi / 2$ corresponding to the boundary.)

in its homology class, namely $\mathfrak{m}_{B}$ and $\mathfrak{m}_{D}=\mathfrak{m}_{A B C}$ in the case of (1.3), or $\mathfrak{m}_{A}$ and $\mathfrak{m}_{C}$ in the case of (1.4). In the following construction we will for definiteness ${ }^{18}$ pick the case

$$
\text { Area }\left[\mathfrak{m}_{B}\right]+\text { Area }\left[\mathfrak{m}_{D}\right] \geq \text { Area }\left[\mathfrak{m}_{A}\right]+\text { Area }\left[\mathfrak{m}_{C}\right]
$$

so that $S(A C)=S(A)+S(C)$. (Although this implies $I(A: C)=0$, we will develop our proof of MMI without the reliance on this fact.)

Converting the above statements to the bit thread description [12, 13], the entanglement entropy of each region is given by the flux of the corresponding maximizer flow. Recall that any maximizer flow for the region $A$ is then guaranteed to be maximally packed (i.e. $\left|v_{A}\right|=1$ ) on $\mathfrak{m}_{A}$, and similarly for the regions $B, C$, and $A B C$, which are maximally packed on the corresponding minimal surfaces. Elsewhere these individual flows admit some floppiness, and at the AdS boundary they are maximally floppy. Hence there is a large amount of freedom in picking the maximizer flows $v_{A}, v_{B}, v_{C}$, and $v_{A B C}$. However, as explained above, a generic choice would not necessarily satisfy the flow conditions (2.2) for the $\tilde{v}_{i}$ 's defined in (2.6); our task is to find an explicit construction which would guarantee that $\left|\tilde{v}_{1}\right| \leq 1,\left|\tilde{v}_{2}\right| \leq 1$, and $\left|\tilde{v}_{3}\right| \leq 1$ simultaneously throughout the bulk.

To build up some intuition, we first take a short detour to illustrate why constructing flows is not trivial, and in particular requires some global considerations. We will however start in section 3.2 with an observation that the set up indicated in section 3.1 actually contains a hidden simplification, which we could use to prove the desired result in this particular case. We will then explain in section 3.3 why this method is not amenable to an easy generalization, and motivate the general construction, which will simultaneously

\footnotetext{
${ }^{18}$ As we further explain in footnote 26 , this is not a limitation since an analogous method will work for the opposite sign of the inequality in (3.2) as well. Moreover, MMI is preserved under purification and permutations, so the choice (3.2) merely fixes a labeling convention.
} 
reveal the connection to bulk locality. To skip to the construction itself, the reader may proceed directly to section 3.4.

\subsection{Cooperative flows for uncorrelated regions}

In encountering the requisite expressions (2.6), one might be tempted to try reducing each line to only two terms by canceling the other two against each other. Unfortunately, we can not set, say, $v_{A}=v_{B}$, since by subadditivity, such flow across $\mathfrak{m}_{A}$ and $\mathfrak{m}_{B}$ would exceed the common bottleneck $\mathfrak{m}_{A B},{ }^{19}$ and similarly for the other adjoining regions. The only simultaneous flows we are allowed a-priori (without additional constraints), are the ones corresponding to nested regions, i.e. $A B C$ and (at most) one of $A, B$, or $C$. This however ensures the norm bound for only one of the $\tilde{v}_{i}$ 's. To deal with the other two $\tilde{v}_{i}$ 's, we need further cancellations.

In fact, one sufficient such cancellation would be easy to achieve in the case where some pair of regions has vanishing mutual information, namely when $I(A: C)=0$ or $I(B: D)=0$. In the former case, we can find a common maximizer flow for both regions by channeling the flow from ${ }^{20} A$ into $B D$ but not $C$, and similarly channeling the flow from $C$ into $B D$ but not $A$. In particular, when subadditivity for $A$ and $C$ is saturated, the flow bottleneck for $A C$ coincides with the union of the individual bottlenecks for $A$ and for $C$, which means that any maximizer flow $v_{A C}$ in fact saturates the norm bound on $\mathfrak{m}_{A}$ and on $\mathfrak{m}_{C}$, so that it is by definition simultaneously a maximizer flow for these individual regions, i.e., $v_{A C}=v_{A}=v_{C}$. In such a case, using additionally the nesting of $B$ and $A B C$ to set $v_{A B C}=v_{B}$, the expressions in (2.6) would simplify to

$$
\tilde{v}_{1}=v_{B}, \quad \tilde{v}_{2}=v_{B}, \quad \tilde{v}_{3}=v_{A} \quad(\text { if } I(A: C)=0)
$$

which means that all the $\tilde{v}_{i}$ 's are manifestly flows.

In the alternate case of $I(B: D)=0$, we can similarly set $v_{B}=v_{D}=-v_{A B C}$. Having used up $v_{A B C}$, one might worry that we can no longer use nesting to get a further simplification; however, as one can see from figure 1, this case was in fact just a rotated/relabeled version of the previous case, so the same arguments should still go through. And indeed, we still do have a form of nesting: $A \subset A B D$, which along with purity $\overline{A B D}=C$ can be used to set $v_{A}=v_{A B D}=-v_{C}$. In this case, (2.6) would simplify to

$$
\tilde{v}_{1}=v_{A}, \quad \tilde{v}_{2}=-v_{A}, \quad \tilde{v}_{3}=-v_{B} \quad(\text { if } I(B: D)=0)
$$

so once again, all the $\tilde{v}_{i}$ 's are manifestly flows.

One might at this point be tempted to declare victory: after all, (3.1) implies that at least one of $I(A: C)$ and $I(B: D)$ vanishes, since if (3.2) holds, then $S(A C)=S(A)+S(C)$, whereas for the opposite sign of the inequality, $S(B D)=S(A C)=S(B)+S(D)$.

\footnotetext{
${ }^{19}$ This statement holds whenever subadditivity is not saturated (i.e. when $I(A: B)>0$, since then $S(A)+S(B)>S(A B)$ ), and in the present case, the mutual information $I(A: B)$ in fact diverges. (Geometrically this is associated with the UV divergence in the areas of $\mathfrak{m}_{A}$ and $\mathfrak{m}_{B}$ at the common entangling surface $A \cap B$, which is not present for $\mathfrak{m}_{A B}$.)

${ }^{20}$ In the interest of familiarity, here we conform to the convention of [12] in drawing the bit threads for a given region $X$ as directed out of $X$ into the bulk (rather than as spacetime-outward-directed flow used in $[13])$.
} 
However, (3.1) was predicated on the simple setup sketched in figure 1. We can easily have both $I(A: C)$ and $I(B: D)$ non-zero (and even make both diverge) by taking all regions to be pairwise near (or even adjoining). ${ }^{21}$ Since we can generically have both $I(A: C)>0$ and $I(B: D)>0$, the previous argument using (3.3) or (3.4) would not apply. On the other hand, we can still use nesting along with purification in full generality: e.g., we could use nesting of $B \subset A B C$ to set $v_{B}=v_{A B C}$, and use nesting of $A \subset A B D=\bar{C}$ to set $v_{A}=-v_{C}$. Unfortunately, substituting these into (2.6) gives

$$
\tilde{v}_{1}=v_{B}+v_{A}, \quad \tilde{v}_{2}=v_{B}-v_{A}, \quad \tilde{v}_{3}=0,
$$

so although we can achieve full cancelation in one of the $\tilde{v}_{i}$ 's, the other two fail to be flows since they exceed the norm bound. Hence we need to find a more general method of constructing the requisite maximizer flows.

\subsection{Motivation for our strategy}

To motivate such a construction, let us then first consider the structure of a generic flow. Since each bit thread has two endpoints, located somewhere on the boundary (i.e. within either $A$ or $B$ or $C$ or $D$ ), we can think of a most general flow as composed out of (a subset of) thread bundles joining all possible pairs of regions. In particular, in general we could have bundles connecting $A \leftrightarrow B, A \leftrightarrow C, A \leftrightarrow D, B \leftrightarrow C, B \leftrightarrow D$, and $C \leftrightarrow D$. Being a piece of a flow, each thread bundle individually satisfies the flow conditions (2.2), and if we're decomposing a single flow, these pieces manifestly cannot overlap. On the other hand, viewed as more abstract building blocks for a collection of flows, we could in general consider overlapping thread bundles, as long as they uphold the total norm bound. ${ }^{22}$ To use the thread bundles as building blocks of a flow for a given region $X$, we simply consider all thread bundles emanating from $X .^{23}$

So far we have merely refined the nomenclature for a given flow, but we will now explain the utility of considering such a decomposition. Recall that flows for non-nested but correlated regions $v_{A}$ and $v_{B}$ are incompatible. This however does not necessarily mean that their individual thread bundles are likewise. In particular, one can envision constructing all four maximizer flows, $v_{A}, v_{B}, v_{C}$, and $v_{D}=-v_{A B C}$ out of the same set of pre-specified thread bundles, by picking their orientations appropriately. For example the bundle $A \leftrightarrow B$ would contribute to $v_{A}$ in the orientation $v_{A \rightarrow B}$ and to $v_{B}$ in the opposite orientation $v_{B \rightarrow A}=-v_{A \rightarrow B}$. (Note that by purity and nesting, we can always use the same set of threads to obtain flows for two disjiont regions $X$ and $Y \subset \bar{X}$ by taking $v_{X}=-v_{\bar{X}}=-v_{Y}$.) As is intuitively clear and easy to check explicitly, keeping all the

\footnotetext{
${ }^{21}$ In the $\mathrm{AdS}_{3}$ case, this can be achieved by having some of the regions be composed of multiple intervals (as exemplified in figure 7), while in higher dimensions, this is possible even with a single simply-connected region per subsystem.

${ }^{22}$ In terminology of [14], such objects are dubbed 'multiflows'. In the present work (unlike [14]), we will in fact construct thread bundles which do not overlap.

${ }^{23}$ We could optionally also include other thread bundles connecting pairs of regions distinct from $X$ (and not interfering with $X$ 's bundles), but since these are irrelevant for computing $S(X)$, we can choose to ignore them.
} 


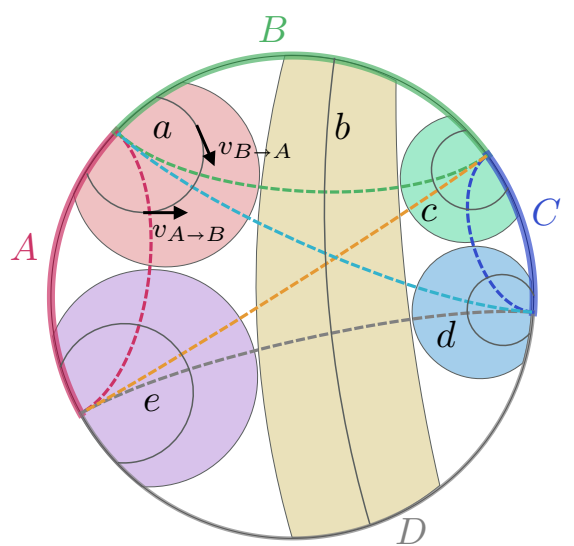

(a)

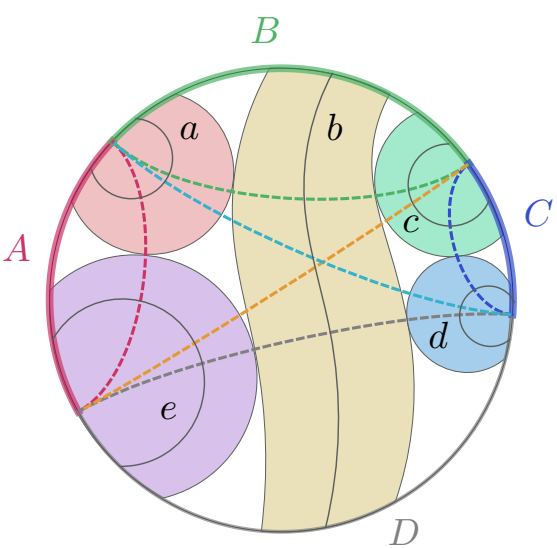

(b)

Figure 2. Separate components (thread bundles) of the maximizer flows $v_{A}, v_{B}, v_{C}$, and $v_{D}$, passing through separate parts of the corresponding minimal surfaces as indicated, though only a single thread for each bundle is shown explicitly. Each thread bundle is confined to its own spatial region, which we compactly label $a$ (for $A \leftrightarrow B$ bundle), $b$ (for $B \leftrightarrow D$ bundle), $c$ (for $B \leftrightarrow C$ bundle), $d$ (for $C \leftrightarrow D$ bundle), and $e$ (for $A \leftrightarrow D$ bundle). The two panels illustrate a residual 1-parameter freedom in the construction, further discussed in section 3.4.1. (a) In the $a$ bundle, the arrows indicate that the same thread can be used in both directions, labeled $v_{A \rightarrow B}$ and $v_{B \rightarrow A}$. (b) The relative sizes of the thread bundles are not fixed; one can vary them at the expense of e.g. bending the $b$ flow.

thread bundles spatially disjoint from each other would then guarantee that each of $v_{A}, v_{B}$, $v_{C}$, and $v_{D}$ constructed from them is likewise a flow. Moreover, each is a maximizer flow if the thread bundles collectively render each of $\mathfrak{m}_{A}, \mathfrak{m}_{B}, \mathfrak{m}_{C}$, and $\mathfrak{m}_{D}$ to be everywhere crossed perpendicularly by some bundle (with the correct orientation) with unit norm.

The most economical way to construct disjoint thread bundles is to require that they in fact saturate the norm bound everywhere within their domain of support, since accommodating a smaller norm for any bundle would require greater bulk volume. In other words, we wish to comb the flow configuration into maximally collimated thread bundles, in such a way that these bundles do not intersect each other. Consequently, for our configurations (cf. figure 1), the presence of an $A \leftrightarrow C$ bundle is incompatible with simultaneous presence of a $B \leftrightarrow D$ bundle. This is however not a problem, since as explained above, $I(A: C)=0$ obviates the need for the $A \leftrightarrow C$ bundle. ${ }^{24}$ This reasoning motivates us to try constructing a thread bundle configuration of the form sketched in figure 2, which exemplifies how each maximizer flow can be partitioned into several thread bundles, each remaining within its associated bulk region; for compactness of notation we label these $a, b, c, d, e$ as indicated in figure 2.

Once we have constructed such a set of cooperative thread bundles from which we can assemble all four maximizer flows, it follows almost immediately that their linear

\footnotetext{
${ }^{24}$ This is manifest by choosing $v_{A}=v_{C}$, but our construction will actually be more thrifty, and have no overlap at all between the two flows (i.e., implement $\left|v_{A}\right|\left|v_{c}\right|=0$ everywhere). Similarly, the alternate case $I(B: D)=0$ would obviate the need for the $B \leftrightarrow D$ thread bundle.
} 


\begin{tabular}{|c|c|c|c|c|c|}
\hline & $a$ & $b$ & $c$ & $d$ & $e$ \\
\hline$v_{A}$ & $v_{A \rightarrow B}$ & 0 & 0 & 0 & $v_{A \rightarrow D}$ \\
\hline$v_{B}$ & $v_{B \rightarrow A}$ & $v_{B \rightarrow D}$ & $v_{B \rightarrow C}$ & 0 & 0 \\
\hline$v_{C}$ & 0 & 0 & $v_{C \rightarrow B}$ & $v_{C \rightarrow D}$ & 0 \\
\hline$v_{A B C}$ & 0 & $v_{B \rightarrow D}$ & 0 & $v_{C \rightarrow D}$ & $v_{A \rightarrow D}$ \\
\hline
\end{tabular}

Table 1. Partitioning of the 4 maximizer flows (indicated in the left column) into thread bundles confined to the 5 bulk regions (indicated in the top row). Each row corresponds to an equation delineating this partition, e.g. the first row gives $v_{A}=v_{A \rightarrow B}+v_{A \rightarrow D}$, where the two r.h.s. terms are supported entirely within the regions $a$ and $e$, respectively, and similarly for the other rows.

\begin{tabular}{|c|c|c|c|c|c|}
\hline & $a$ & $b$ & $c$ & $d$ & $e$ \\
\hline$\tilde{v}_{1}$ & 0 & $v_{B \rightarrow D}$ & $v_{B \rightarrow C}$ & 0 & $v_{A \rightarrow D}$ \\
\hline$\tilde{v}_{2}$ & $v_{B \rightarrow A}$ & $v_{B \rightarrow D}$ & 0 & $v_{C \rightarrow D}$ & 0 \\
\hline$\tilde{v}_{3}$ & $v_{A \rightarrow B}$ & 0 & $v_{C \rightarrow B}$ & $v_{C \rightarrow D}$ & $v_{A \rightarrow D}$ \\
\hline
\end{tabular}

Table 2. Explicit check that the $\tilde{v}_{i}$ 's defined by (2.6) are indeed flows, given the partition in table 1 . Each row gives an equation with l.h.s. being the first column and r.h.s. the sum of the terms in the remaining columns, as in table 1 . The columns correspond to the distinct bulk regions (cf. figure 2) and we see that in each region, $\tilde{v}_{i}$ is manifestly a flow.

combinations $\tilde{v}_{2}, \tilde{v}_{3}$, and $\tilde{v}_{1}$ defined via (2.6) will likewise be flows. The crux of our MMI proof then boils down to showing that a configuration envisioned in figure 2 is indeed always tenable, which we will now demonstrate by an explicit construction.

\subsection{Flow construction}

In this section we present an explicit construction for the maximizer flows $v_{A}, v_{B}, v_{C}$, and $v_{A B C}$ which render the $\tilde{v}_{i}$ 's of (2.6) likewise flows.

Starting with partitioning of $\mathrm{AdS}_{3}$ boundary time slice of the type sketched in figure 1, we first show that we can partition the maximizer flows as indicated in table 1 (cf. figure 2). In particular, in section 3.4.1 we argue that we can comb the maximizer flow from $A$ into a thread bundle ${ }^{25} v_{A \rightarrow B}$ leading to $B$ and a thread bundle $v_{A \rightarrow D}$ leading to $D$ (but not an $v_{A \rightarrow C}$ thread bundle thanks to (3.2)), and similarly for $B, C$, and $D$, such that the thread bundles pass only through restricted disjoint bulk regions (denoted $a, b, c, d, e$ ).

Once we show (in the remainder of this section) that such a partition is possible, the rest is straightforward: applying the definition (2.6), we can directly evaluate the $\tilde{v}_{i}$ 's, and examine them within each region. The result is packaged in table 2 , which gives the explicit contributions within each region. The crucial observation here is that each entry in the table is of the form $v_{X \rightarrow Y}$ (i.e. a bundle of a single flow $v_{X}$ and therefore manifestly a flow).

\footnotetext{
${ }^{25}$ Recall that for $X, Y$ chosen from the fundamental regions $A, B, C$, and $D$, the notation $v_{X \rightarrow Y}$ simply indicates the part of the $v_{X}$ which flows into $Y$. The only requirement that we impose on such a partitioning is that $v_{X \rightarrow Y}=-v_{Y \rightarrow X}$ for all $X, Y$. Since this corresponds to merely reversing the orientation of the bit threads, both flows have the same norm and divergence.
} 
Hence even though each of the $\tilde{v}_{i}$ 's is the sum of three bulk flows, the result is nevertheless of unit-bounded norm everywhere. This establishes that $\tilde{v}_{i}=v_{i}$ (i.e. each $\tilde{v}_{i}$ is a flow) as advertised. ${ }^{26}$

\subsubsection{Maximizer flow partitions}

We now explain why the partition indicated in table 1 and exemplified in figure 2 is indeed always possible. We have already seen that the decomposition summarized in the rows of table 1 without requiring the bulk regions $a, b, c, d$, and $e$ to be disjoint, is necessitated by the divergencelessness condition (2.2) (since each thread from a given region has to end in one of the other regions), along with the observation that we need not activate any thread bundles for uncorrelated regions. What remains to be shown is the compatibility between these respective partitions, namely that the thread bundles are mutually non-overlapping throughout the bulk. Since a general maximizer flow from each region necessarily saturates the norm bound on the associated minimal surface presenting a bottleneck for the flow, the first step is to verify the consistency of partitioning on the corresponding minimal surfaces.

In the present case, the surface $\mathfrak{m}_{A}$ splits into two parts, $\mathfrak{m}_{A}^{a}$ and $\mathfrak{m}_{A}^{e}$, associated with the thread bundles $\left(a\right.$ and $e$ ) ending on $B$ and $D$, respectively. Similarly, $\mathfrak{m}_{B}$ splits into three parts, $\mathfrak{m}_{B}^{a}, \mathfrak{m}_{B}^{b}$, and $\mathfrak{m}_{B}^{c}$, channeling the bundles $(a, b, c)$ from $B$ into $A, D$, and $C$, respectively, and so on. Moreover, since the threads of $v_{X \rightarrow Y}$ through $\mathfrak{m}_{X}^{z}$ coincide with the threads of $v_{Y \rightarrow X}$ through $\mathfrak{m}_{Y}^{z}$, but with opposite orientation, the areas of the two minimal surface parts must likewise match, Area $\left[\mathfrak{m}_{X}^{z}\right]=$ Area $\left[\mathfrak{m}_{Y}^{z}\right]$, for all $X, Y \in A, B, C, D$ and corresponding $z \in a, b, c, d, e$.

Written out explicitly, given $\mathfrak{m}_{A}, \mathfrak{m}_{B}, \mathfrak{m}_{C}$, and $\mathfrak{m}_{D}$, the minimal surface subdivisions need to satisfy the following constraints:

$$
\begin{aligned}
\text { Area }\left[\mathfrak{m}_{A}\right] & =\text { Area }\left[\mathfrak{m}_{A}^{a}\right]+\text { Area }\left[\mathfrak{m}_{A}^{e}\right] \\
\text { Area }\left[\mathfrak{m}_{B}\right] & =\text { Area }\left[\mathfrak{m}_{B}^{a}\right]+\text { Area }\left[\mathfrak{m}_{B}^{b}\right]+\text { Area }\left[\mathfrak{m}_{B}^{c}\right] \\
\text { Area }\left[\mathfrak{m}_{C}\right] & =\text { Area }\left[\mathfrak{m}_{C}^{c}\right]+\text { Area }\left[\mathfrak{m}_{C}^{d}\right] \\
\text { Area }\left[\mathfrak{m}_{D}\right] & =\text { Area }\left[\mathfrak{m}_{D}^{e}\right]+\text { Area }\left[\mathfrak{m}_{D}^{b}\right]+\text { Area }\left[\mathfrak{m}_{D}^{d}\right] \\
\text { Area }\left[\mathfrak{m}_{A}^{a}\right] & =\text { Area }\left[\mathfrak{m}_{B}^{a}\right] \\
\text { Area }\left[\mathfrak{m}_{B}^{b}\right] & =\text { Area }\left[\mathfrak{m}_{D}^{b}\right] \\
\text { Area }\left[\mathfrak{m}_{B}^{c}\right] & =\text { Area }\left[\mathfrak{m}_{C}^{c}\right] \\
\text { Area }\left[\mathfrak{m}_{C}^{d}\right] & =\text { Area }\left[\mathfrak{m}_{D}^{d}\right] \\
\text { Area }\left[\mathfrak{m}_{A}^{e}\right] & =\text { Area }\left[\mathfrak{m}_{D}^{e}\right]
\end{aligned}
$$

\footnotetext{
${ }^{26}$ Recall that the above construction assumed that $I(A: C)=0$ which implies (3.2). However, as explained in footnote 18, this was merely a notational convention. If $I(A: C)>0$ so that the $B D$ flows have more stringent bottleneck than the $A C$ system, we can simply reconfigure the thread bundles so as to have a component $v_{A \rightarrow C}$ instead of $v_{B \rightarrow D}$, along with the corresponding rearrangement of the regions $a, b, c, d, e$ (such that $a$ adjoins $c, d$ adjoins $e$, and $b$ passes between $a$ and $e$ and between $c$ and $d$ ). Consequently in table 2 , the $b$ column would contain $v_{A \rightarrow C}$ and $v_{C \rightarrow A}$ in the first and 2nd rows respectively, while the rest of the table remains identical.
} 
This constitutes 9 equations for 10 unknowns, and therefore we expect to have a 1parameter family of solutions, as illustrated in figure $2 \mathrm{a}$ and figure $2 \mathrm{~b}$. One procedure of constructing a partitioning is as follows. Choose the partition of $\mathfrak{m}_{A}$ (in the present case that is just a single point $p_{A}$ ). This determines $\mathfrak{m}_{A}^{a}$ and $\mathfrak{m}_{A}^{e}$, and hence also $\mathfrak{m}_{B}^{a}$ and $\mathfrak{m}_{D}^{e}$. Now find the partition of $\mathfrak{m}_{C}$ (determined by a point $p_{C}$, which immediately specifies $\mathfrak{m}_{C}^{c}, \mathfrak{m}_{B}^{c}, \mathfrak{m}_{C}^{d}$, and $\mathfrak{m}_{D}^{d}$ ) so as to satisfy the remaining equation of (3.6), namely Area $\left[\mathfrak{m}_{B}^{b}\right]=$ Area $\left[\mathfrak{m}_{D}^{b}\right]$. Since if we slide $p_{C}$ sufficiently "up" along $\mathfrak{m}_{C}$ towards $B$, we close off $\mathfrak{m}_{D}^{b}$, whereas if we slide $p_{C}$ "down" towards $D$, we close off $\mathfrak{m}_{B}^{b}$, a solution clearly exists where the two terms become equal. This completes the argument that minimal surface partition envisioned in figure 2, i.e. satisfying (3.6), always exists.

Now that we have confirmed that a viable partitioning of the 4 minimal surfaces exits, we discuss the procedure for constructing the full maximizer flow for each region. There is of course large freedom in how we construct the requisite maximizer flows; here we present two particularly simple constructions which are easy to generalize. Although the flows are required to saturate the norm bound only on the associated minimal surface, we will find it convenient to construct them so as to remain maximally packed (and therefore equidistant) everywhere, since this utilizes the bulk geometry most economically. Geometrically, such a construction is actually quite simple, and is based on the observation explained in [13] that a foliation of a bulk region by minimal surfaces induces a maximally-collimated flow. Intuitively this is because the minimal surfaces have by definition vanishing expansion for the normal congruence (i.e., vanishing extrinsic curvature), so the bit threads which pass perpendicularly through any leaf of such foliation locally cannot change their cross-sectional density; in other words, they must remain maximally packed across any minimal surface they cross perpendicularly. ${ }^{27}$

\subsubsection{Foliation building blocks}

One strategy to obtain maximally packed thread bundle, then, is to start by considering a family of minimal surfaces which foliate the bulk space, since the normal congruence to these surfaces describes a flow. In the present case of 2-dimensional Poincare disk geometry, the foliating surfaces are simply geodesics, which indeed foliate the space as long as their endpoints vary continuously in any manner which generates a boundary foliation (which we can think of as a family of nested intervals whose endpoints collectively cover the boundary). ${ }^{28}$ Labeling each leaf of the foliation by a parameter $\lambda \in[0,1]$, we can

\footnotetext{
${ }^{27}$ One might also naively worry that the flows encounter other bottlenecks which we have not taken into account. For example the $b$ thread bundle crosses both $\mathfrak{m}_{A B}$ and $\mathfrak{m}_{B C}$ (cf. figure 2), which a-priori need not have larger area than, say, $\mathfrak{m}_{B}$. However, this is not a problem (as we explicitly demonstrate below), since the non-perpendicularly-crossed surfaces have by construction smaller thread density and therefore larger area.

${ }^{28}$ Recall that bulk minimal surfaces (here geodesics) anchored on nested regions (here intervals) are guaranteed not to intersect (since doing so would contradict global minimality), and the AdS geometry guarantees that there is a unique geodesic for each boundary interval and that a geodesic through any point and in any direction has both its endpoints on the boundary. The latter two properties do not hold universally for arbitrary static asymptotically AdS geometries, though they do hold robustly when the
} 


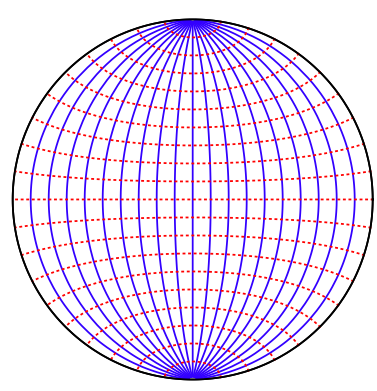

(a)

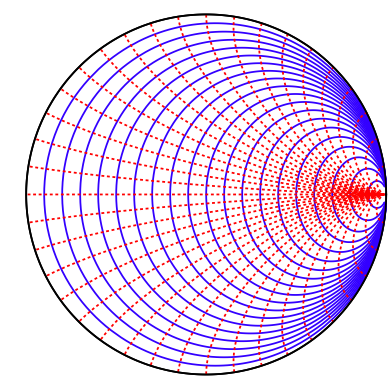

(b)

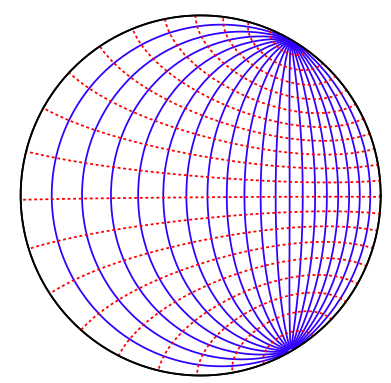

(c)

Figure 3. Examples of flows (solid blue curves) induced by minimal surface foliations (dotted red curves). (While we use the coordinates of figure 1, we don't maintain constant proper spacing between the treads - doing so would clump them too much near the boundary.) Such foliations are specified by the endpoints $\varphi$ of the bounding interval, as functions of $\lambda \in[0,1]$ (see text for details). In particular, we illustrate foliations generating (a) antipodal threads, (b) threads with both endpoints pinned at a single point of the Poincare disk boundary, and (c) threads with endpoints pinned at generically-separated points.

specify the foliation by two functions, $\varphi^{L}(\lambda)$ and $\varphi^{R}(\lambda)$, corresponding to the angular position of the two endpoints of the boundary interval. A foliation requires each function to be monotonic, but in opposite directions (so as to get nested intervals), such that $\varphi^{L}(0)=\varphi^{R}(0)$ and $\varphi^{L}(1)=\varphi^{R}(1)+2 \pi$.

We sketch some simple examples in figure 3 . In figure $3 \mathrm{a}$, the endpoints vary symmetrically, e.g. $\varphi^{L, R}(\lambda)=\frac{\pi}{2} \pm \pi \lambda$. In this case the threads happen to coincide with constant-time projections of null geodesics through AdS. In figure 3b, we fix one endpoint and let the other span the rest of the boundary, $\varphi^{R}(\lambda)=0, \varphi^{L}(\lambda)=2 \pi \lambda$. In this case the threads happen to be horocycles on the Poincare disk. Although this looks like all the bit threads start and end at the same point, this is merely due to the conformal rescaling. The flow lines actually follow constant- $z$ contours in Poincare coordinates $d s^{2}=\left(-d t^{2}+d x^{2}+d z^{2}\right) / z^{2}$ (they are generated by geodesics at constant $x$ ), where it is manifest that they straddle the $x=-\infty$ and $x=+\infty$ parts of the boundary. ${ }^{29}$ One can likewise separate the flow ends, as shown in figure 3c. For example for the endpoints given by $\varphi^{R}(\lambda)=\varphi_{0}-2 \varphi_{0} \lambda$ and $\varphi^{L}(\lambda)=\varphi_{0}+\left(2 \pi-2 \varphi_{0}\right) \lambda$, we see that this case interpolates between the first two. Of course, one can also have more irregular flows, and in fact all these flows can be patched together across any common minimal surface (such at the horizontal bisectors in figure 3 ).

As we see, we can comb the flows in a large (continuously infinite) variety of ways, and from any boundary point to any other boundary point. However, such foliations cannot admit multiple thread bundles, namely multiple starting and ending points for a given flow. To incorporate this more general case, we have to use multiple foliations simultaneously,

deformations from pure AdS are not too large. We revisit the more general case in section 4.2.

${ }^{29}$ One can also resolve this confluence by working at finite cutoff, where we could either only require the endpoints to get within the cutoff scale from each other (e.g. $\varphi^{L}(0)=\varepsilon$ and $\varphi^{L}(1)=2 \pi-\varepsilon$ ), or determine the endpoints by cutting off the same set of spacelike geodesics at finite radius (in which case both endpoints would vary). 


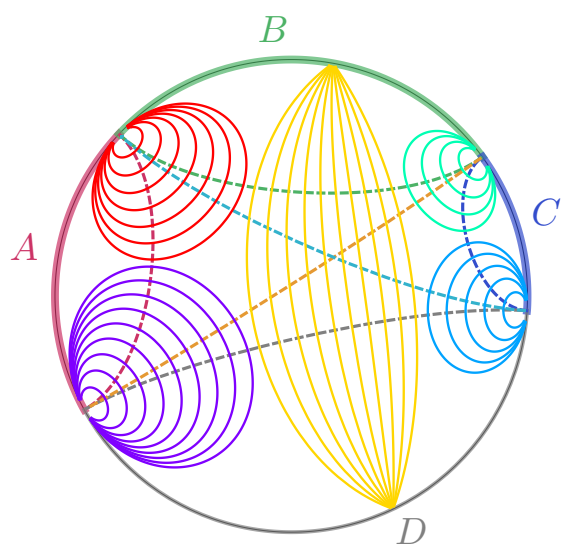

(a)

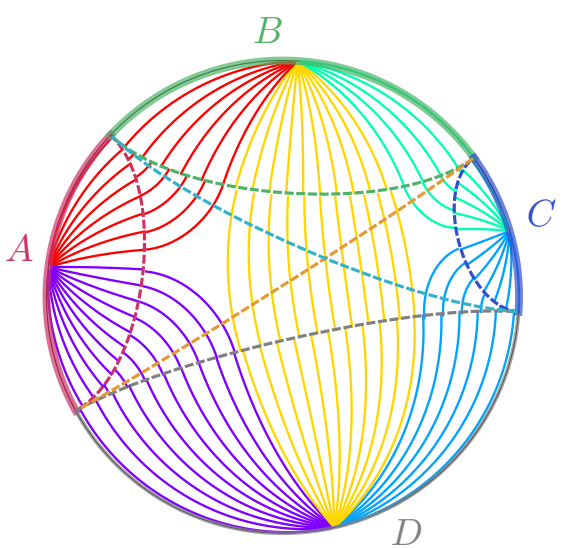

(b)

Figure 4. Illustration of two distinct constructions for obtaining combed maximizer flows using foliations. (a) smooth flows obtained by 5 superimposed foliations. (b) patched flows obtained by a single bulk 'local foliation'. The boundary regions and associated minimal surfaces are as in figure 1, and the threads are indicated by solid curves, with distinct colors delineating the regions $a, b, c, d, e$ of figure 2 which contain the corresponding thread bundles.

or generalize the foliating surfaces to a more localized construct. As an example of each, we consider two particularly natural constructions, shown in figure 4 .

1. Use a complete smooth foliation (by entire minimal surfaces) for each thread bundle. This leads to all threads being smooth, but a given foliation is 'active' (in terms of guiding a thread) in only a subset of the bulk. Hence there are multiple simultaneous bulk foliations. See section 3.4.3 below for explicit construction and figure 4a for the resulting plot.

2. Use a single bulk foliation for (almost) the entire bulk, but by only piecewise-minimal surfaces. The resulting flows are typically not smooth. See section 3.4.4 for explicit construction and figure $4 \mathrm{~b}$ for the corresponding plot.

\subsubsection{Foliation for each thread bundle, smooth flows}

We first consider the case with full bulk foliation per bundle. Since we have 5 thread bundles confined to 5 separate bulk regions $a, b, c, d, e$, we will have 5 distinct foliations. Each foliation is specified by a family of minimal surfaces (geodesics) characterized by their endpoints $\varphi^{L}(\lambda)$ and $\varphi^{R}(\lambda)$ for $\lambda \in[0,1]$, as indicated in figure 5. For the $a, c, d$, and $e$ bundles (defining the associated bulk regions), the foliations are all of the horocycle type sketched in figure $3 \mathrm{~b}$ (rotated in global coordinates): the minimal surfaces 'fan out' from one endpoint, say $\varphi^{L}$, with the other endpoint $\varphi^{R}$ spanning the rest of the boundary, $\varphi^{R}(\lambda)=\varphi^{L}+2 \pi \lambda$. These families then contain the minimal surfaces with the given fixed endpoint: the $a$ family, shown in figure $5 \mathrm{a}$, in particular contains $\mathfrak{m}_{A}$ and $\mathfrak{m}_{B}$ (as well as $\mathfrak{m}_{B C}$ ), the $c$ family contains $\mathfrak{m}_{B}$ and $\mathfrak{m}_{C}$, and so forth. The final family, $b$, indicated in figure $5 \mathrm{~b}$, is of the type sketched in figure $3 \mathrm{c}$, varying both endpoints so as to contain $\mathfrak{m}_{B}$ 


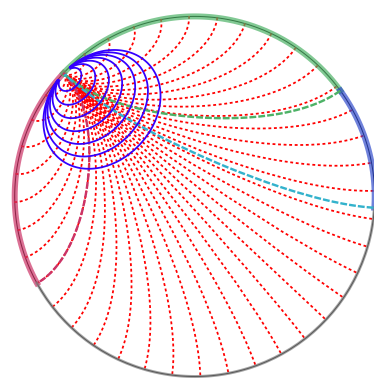

(a)

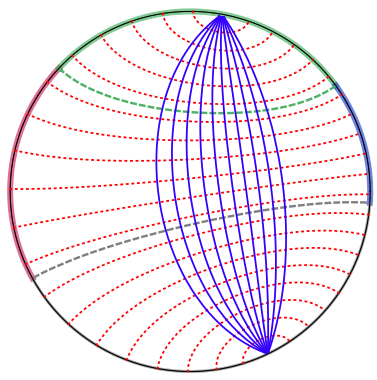

(b)

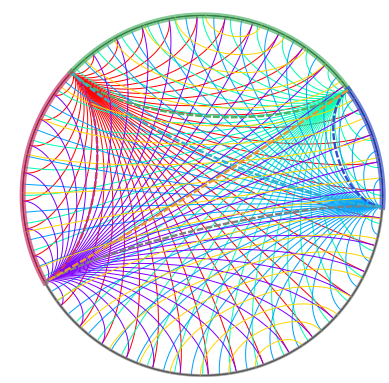

(c)

Figure 5. Specific foliations which generate the smooth flow configuration indicated in figure 4a, in particular generating (a) the flow $v_{A \rightarrow B}$ and (b) the flow $v_{B \rightarrow D}$. When suitably restricted (as indicated by the solid blue curves) these give the thread bundles $a$ and $b$, respectively. (c) All 5 foliations (color-coded as in figure 2) superposed on each other.

and $\mathfrak{m}_{D} \cdot{ }^{30}$ In figure $5 \mathrm{c}$ we superpose all five foliations (without including the corresponding threads), to show how are the individual leaves of the foliations related to each other.

Since for a given thread bundle only a part of its corresponding foliation is used, our remaining task is to show that the utilized regions are mutually compatible. In particular for the $v_{A \rightarrow B}$ flow associated to the bulk region a (figure $5 \mathrm{a}$ ), we only use the foliating minimal surfaces for normal congruence passing through $\mathfrak{m}_{A \rightarrow B}$ and $\mathfrak{m}_{B \rightarrow A}$ as indicated, and similarly for the other thread bundles. In section 3.4.1 we have argued that the minimal surfaces $\mathfrak{m}_{A}, \mathfrak{m}_{B}, \mathfrak{m}_{C}$, and $\mathfrak{m}_{D}$ can be partitioned in a compatible fashion, i.e. satisfying the bottleneck equations (3.6). Hence what remains is to show that the rest of the bundles can likewise accommodate themselves so that distinct thread bundles don't overlap each other.

Let us consider, say, the compatibility of $a$ and $b$ flows (the arguments for the compatibility of the other flows being identical). We want to show that $v_{B \rightarrow A}$ and $v_{B \rightarrow D}$ do not intersect. The most 'worrisome' regions are where the thread bundles approach closest to each other, i.e. just around the minimal surface $\mathfrak{m}_{B}$. Both flows are maximally constrained at $\mathfrak{m}_{B}$, and they pass normally through it (consistently with $\mathfrak{m}_{B}$ belonging to both foliation families, $a$ and $b$, cf. figure $5 \mathrm{a}$ and figure $5 \mathrm{~b}$ ). Since at $\mathfrak{m}_{B}$ itself we ensured that the flows are compatible by the $\mathfrak{m}_{B \rightarrow A}, \mathfrak{m}_{B \rightarrow D}$ partitioning, to see that subsequently the thread bundles bend away from each other, let us consider a leaf of the $a$ and $b$ foliations which have a common right endpoint $\varphi_{a}^{R}\left(\lambda_{a}\right)=\varphi_{b}^{R}\left(\lambda_{b}\right)=p_{\beta}+\epsilon$, just 'above' $\mathfrak{m}_{B}$. The corresponding left endpoints are distinct: $\varphi_{a}^{L}\left(\lambda_{a}\right)=p_{\alpha}$, while $\varphi_{b}^{R}\left(\lambda_{b}\right)=p_{\alpha}-\epsilon^{\prime}$, with the $b$-leaf's defining interval lying within the $a$-leaf's interval (as the reader may try to verify by a careful look at figure 5c). This means that the $a$ leaf bends further to the left than the $b$ leaf (recall that the leafs cannot intersect since otherwise they would contradict the assumption of

\footnotetext{
${ }^{30}$ While we saw that the 'bottleneck' equations (3.6) in general have a 1-parameter family of solutions (illustrated in the two panels of figure 2), in the present case that parameter is used up by choosing $v_{B \rightarrow D}$ to be of the particular form plotted in figure 3c (generated by threads emanating radially outward from a shifted origin in Poincare coordinates and rotated in global coordinates), so that the $B$ and $D$ positions fix this solution uniquely, to the type sketched in figure $2 \mathrm{a}$ as opposed to figure $2 \mathrm{~b}$.
} 
minimality in their construction). Therefore, their normals (which guide their respective flows) likewise bend away from each other: $n_{a}$ (pointing towards $B$ ) more to the left and $n_{b}$ more to the right. We can make a similar argument for the behavior of the flow 'below' $\mathfrak{m}_{B}$ where the flows likewise bend away from each other in the downward direction. This means that the flows remain compatible around their bottleneck $\mathfrak{m}_{B}$.

We can now make a more general argument, applying to the entire remainder of $a$ and $b$ thread bundles of the flow. Consider any bulk point defined by the intersection of the $a$-foliation and $b$-foliation leafs (which, except for points lying on $\mathfrak{m}_{B}$, is uniquely specified by $\lambda_{a}$ and $\lambda_{b}$ ). Inside $B$ 's entangling region, $\varphi_{b}^{L}\left(\lambda_{b}\right)<\varphi_{a}^{L}\left(\lambda_{a}\right)=p_{\alpha}$ and simultaneously, in order for the leafs to intersect, the corresponding intervals cannot be nested, so $\varphi_{b}^{R}\left(\lambda_{b}\right)<$ $\varphi_{a}^{R}\left(\lambda_{a}\right)$. Moreover, the leafs cannot have multiple intersections. This means that at the intersection point, the normal for the $a$ leaf must bend more towards the $A$ region than the normal to the $B$ leaf.

Identical analysis for the remaining places where the thread bundles touch on one of the minimal surfaces then shows that all thread bundles bend away from each other. Namely, once they are compatible on the minimal surfaces (i.e. satisfy the bottleneck equations (3.6)), then they are compatible in the rest of the bulk - in other words they generate cooperative flows.

\subsubsection{Single local foliation for all thread bundles, patched flows}

The previous construction required not just one, but five complete overlapping foliations to argue for cooperation (in particular to verify that the endpoints of foliating geodesics were such as to channel the thread bundles away from each other, cf. figure 5c). Since we ended up using only parts of these foliations, it might seem a bit more economical to consider just a single set of curves within the bulk which would generate all the thread bundles in one go. Of course such a set cannot truly be a foliation in the strict sense that for every bulk point $p$ there exists a unique leaf of the foliation which contains $p$, because the topology of the thread bundles would necessitate some junctions in the leaves. However, what really matters is not that we cover the entire bulk, but rather that the leaves don't intersect. Once we exclude the junctions, this weaker (uniqueness) requirement is certainly possible to satisfy. We will call such partial foliations (which we could think of as foliations of the bulk with some points removed) local foliations.

One (impractical) way to achieve a single local foliation of the bulk would be to take the set of foliations constructed in section 3.4.3, cut them off at their respective thread bundle's edges (which are determined by the minimal surface partitions), and join the leafs through the remaining bulk gaps. However, this offers no particular (either constructive or logical) simplification. It also does not take the opportunity to avoid what is perhaps the most awkward feature of the previous construction, namely that the thread bundles between adjoining regions in section 3.4.3 appear to start and end at the same boundary point. Here we remedy this by a more convenient choice of local foliation, indicated in figure 6 . 


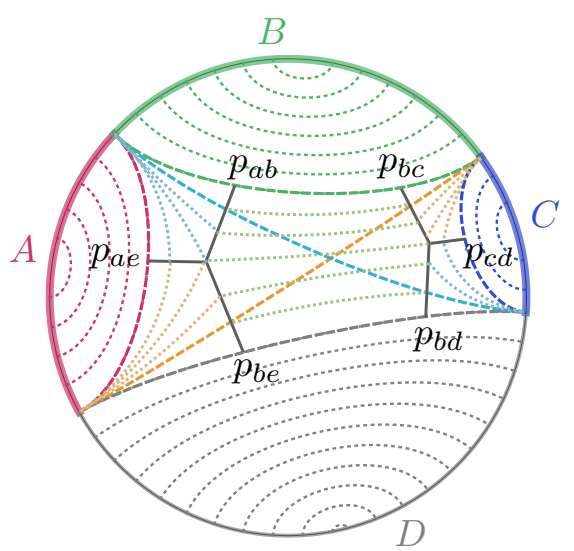

Figure 6. A local foliation designed to obtain the thread bundles indicated in figure $4 \mathrm{~b}$. The 6 black line segments along with the 4 minimal surfaces break up the bulk into 9 different regions, each foliated by its own family of geodesics.

In the homology region ${ }^{31}$ for each of the boundary regions $A, B, C, D$, we use the foliation generated by symmetrically nested boundary intervals as in figure 3a. All thread bundles $v_{X \rightarrow Y}$ emanate from $X$ 's midpoint, and automatically reach the corresponding minimal surface $\mathfrak{m}_{X}$ perpendicularly. On the 4 minimal surfaces we pick a partition satisfying (3.6), which specifies 6 bulk points labeled $p_{a b} \in \mathfrak{m}_{B}, p_{b c} \in \mathfrak{m}_{B}, p_{c d} \in \mathfrak{m}_{C}, p_{b d} \in \mathfrak{m}_{D}$, $p_{b e} \in \mathfrak{m}_{D}$, and $p_{a e} \in \mathfrak{m}_{A}$ as shown in figure 6 (the subscripts indicate where the given thread bundles separate, cf.figure 2). Consider the triangle formed by $p_{a b}, p_{a e}$, and $p_{b e}$, and take its 'center' (to be specified in footnote 32 ) point $p_{a b e}$, and similarly for the $b c d$ junction. Using these $6+2$ points, construct 6 (geodesic) line segments joining the vertices of each triangle to its center point. These segments partition the central quadrilateral region (bounded by the 4 minimal surfaces $\mathfrak{m}_{X}$ ) into 5 bulk regions, one for each thread bundle, thus partitioning the full Poincare disk into 9 regions.

To construct the five thread bundles, we proceed analogously to the previous construction, but with the crucial difference that we don't specify these geodesics by their endpoints on the boundary, but rather by their location along these interior line segments. Concretely, the foliation for thread bundle $a$ is given by the family of geodesics between $p_{A B} \equiv \partial A \cap \partial B$ and the interior bulk point $p_{a}^{R}(\lambda)$ lying in the segments $p_{a b} p_{a b e} p_{b e}$, with $\lambda=0$ at $p_{a b}$ and $\lambda=1$ at $p_{b e}$. Since all geodesics 'fan out' from $p_{A B}$, this is part of a foliation of the type indicated in figure $3 \mathrm{~b}$. Moreover, our choice of $p_{\text {abe }}$ guarantees ${ }^{32}$ that the corresponding threads from $\mathfrak{m}_{A \rightarrow B}$ reach $\mathfrak{m}_{B \rightarrow A}$ and vice-versa, without leaving this region.

\footnotetext{
${ }^{31} \mathrm{By}$ 'homology region' for $X$, we mean the bulk region bounded by the given boundary region $X$ and its associated bulk minimal surface $\mathfrak{m}_{X}$, cf. footnote 42 for a more covariant definition.

${ }^{32}$ The central point $p_{a b e}$ is defined as any point which lies within the triangle formed by the threads passing through the three pairs of vertices in the construction of section 3.4.3. (Hence we retain a lot of freedom in this construction.) Since these threads bend away from their tangents at the minimal surfaces $\mathfrak{m}_{X}$, they cannot intersect the line segments extending these tangents (pieces of which correspond to the segments $p_{a b} p_{a b e}$ and $\left.p_{a b e} p_{b e}\right)$.
} 
The other three thread bundles between adjoining regions (namely $c, d$, and $e$ ), are constructed in identical fashion. The remaining bundle, $b$, can actually be generated as a part of the foliation indicated in figure 3c. And by the same argument as above, these threads joining $\mathfrak{m}_{B \rightarrow D}$ and $\mathfrak{m}_{D \rightarrow B}$ cannot leave the hexagonal region $p_{a b} p_{a b e} p_{b e} p_{b d} p_{b c d} p_{b c}$. This finishes the alternate construction of the thread bundles generating a cooperative flow.

Notice that unlike the previous case discussed in section 3.4 .3 which left 8 bulk regions unpopulated by any thread bundle, we now have only two unpopulated regions (the triangles mentioned above). ${ }^{33}$ In fact, we can reduce this further to just a single unpopulated region, by using only two intersecting line segments, $p_{a e} p_{c d}$ and say one joining midpoint of $\mathfrak{m}_{B \rightarrow D}$ to midpoint of $\mathfrak{m}_{D \rightarrow B}$. The $a, c, d$, and $e$ thread bundles would remain as before, but the $b$ thread bundle now gets split into two kinked sub-strands, one adjoining the $a$ and $e$ thread bundles and the other adjoining the $c$ and $d$ thread bundles. The kinks appearing along the $p_{a e} p_{c d}$ segments pose no particular problem (the norm bound is satisfied everywhere) other than the flow field $v_{B \rightarrow D}$ being strictly-speaking undefined at the segment. We can however locally smooth out the corners to avoid this.

The overall lesson is that there are many ways to construct cooperative flows generated by local foliations (using minimal surface segments). The resulting thread bundles are automatically disjoint and maximally collimated. The gaps they leave behind in the bulk have no physical significance - they (as well as precise position of the thread bundles themselves) may be viewed analogously to a gauge choice. On the other hand, the freedom of satisfying the bottleneck equations (e.g. how far along the $\mathfrak{m}_{B}$ surface can the $b$ thread bundle shift) may have a more direct significance in terms of multipartite entanglement (cf. [14]'s interpretation of $-\frac{1}{2} I_{3}$ ) which would be interesting to explore further. It is worth noting that these thread bundles constitute a special case of a multiflow discussed in [14], where the more general $v_{i j}$ 's of [14] in our construction all saturate the norm bound individually in some regions and vanish entirely in others. We therefore provide an explicit construction of a special type of a 'max multiflow' (in [14]'s language).

\section{Generalizations}

In section 3 we constructed cooperative flows in the simplest class of examples: spatial slice of pure $\mathrm{AdS}_{3}$ with boundary partitioning given by 4 adjoining intervals. This starting point was chosen primarily for ease of illustration, and our arguments in fact apply much more broadly. In this section we examine various natural generalizations. These entail considering more general partitions (still restricted to the form $\mathcal{H}=\mathcal{H}_{A} \otimes \mathcal{H}_{B} \otimes \mathcal{H}_{C} \otimes$ $\mathcal{H}_{D}$ relevant for MMI), more general state (which modifies the asymptotically AdS bulk geometry and allows for time dependence), or even a different theory (including changing the number of dimensions and number of background spacetime components on which the field theory lives).

\footnotetext{
${ }^{33}$ The reader might at this point wonder why the latter appears to have manifestly smaller volume (in fact a finite one) than the former (which has infinite volume owing to some of the regions adjoining the boundary), despite the fact that in both cases we kept all our thread bundles maximally collimated. This apparent discrepancy stems from the usual issue of the UV divergences when dealing with the entire bulk spacetime. In particular, the threads of the present construction include those which 'hug' the boundary and therefore utilize greater bulk volume than the threads of section 3.4.3.
} 
We conjecture that the basic construction outlined above can be adapted to any of the above-mentioned situations, describing a generic classical bulk spacetime satisfying physical energy conditions. More specifically:

Conjecture 4.1 In any holographic CFT state whose gravitational dual corresponds to a classical bulk spacetime with entanglement entropy computed by the HRT prescription, a cooperative flow for any boundary spatial partition exists and can be constructed by maximally collimated thread bundles confined to non-intersecting regions of a bulk Cauchy slice.

Rather than attempting a full analysis (which we leave for future work), we provide some basic arguments and simple checks of our conjecture, intended more as a plausibility argument than a proof. Instead of considering the most general case from the outset, we address how our method can be adapted for each of these generalizations individually. In particular, we consider generalizing the number of spatial regions in section 4.1, generalizing the bulk geometry (within a class of time-reversal symmetric asymptotically $\mathrm{AdS}_{3}$ spacetimes) in section 4.2, generalizing to higher dimesions in section 4.3, and finally allowing for genuine time-dependence in section 4.4 .

\subsection{Multiple sub-partitions}

Recall that the case of 4 adjoining intervals $A, B, C$, and $D$ discussed in the previous section has either $I(A: C)=0$ or $I(B: D)=0$, so the more immediate proof of section 3.2 suffices to guarantee the existence of cooperative flows: in particular, we can pick $v_{A}$ and $v_{B}$ to be arbitrary and specify the remaining maximizer flows accordingly, without any need to split them into disjoint thread bundles. We now demonstrate explicitly that in the more general situation with $I(A: C)>0$ and simultaneously $I(B: D)>0$, the 'combed thread bundle' method of section 3.4 indeed applies. We will first briefly outline a specific construction and then indicate what happens in full generality.

The simplest type of configuration which has all pairwise mutual informations nonvanishing is shown in figure 7a. Here we take the $B D$ system to be larger than the $A C$ one, automatically implementing $I(B: D)>0$. We also take $A=A_{1} \cup A_{2}$ with $A_{2}$ adjoining $C$, so that $I(A: C) \geq I\left(A_{2}, C\right)=\infty$ (where the inequality follows by monotonicity of mutual information (SSA), and the r.h.s. is the usual property of adjoining regions). This means that in decomposing the flows into thread bundles, we will necessarily have $v_{A_{1} \rightarrow B}, v_{A_{1} \rightarrow D}$, $v_{A_{2} \rightarrow B}, v_{A_{2} \rightarrow C}, v_{C \rightarrow D}$, as well as $v_{B \rightarrow D}$ in order to accommodate the non-vanishing mutual information between the corresponding intervals. On the other hand, by the same argument as above, $I\left(A_{1}: A_{2} C\right)=0$ which (again by monotonicity along with non-negativity) implies that $I\left(A_{1}: A_{2}\right)=0$ and $I\left(A_{1}: C\right)=0$. That in turn means that we do not need to turn on the $v_{A_{1} \rightarrow A_{2}}$ or $v_{A_{1} \rightarrow C}$ thread bundles.

There are two additional thread bundles to consider: $v_{A_{2} \rightarrow D}$ and $v_{B \rightarrow C}$, but turning on both of these maximally collimated thread bundles simultaneously would not produce cooperative flows, since the thread bundles necessarily intersect and therefore in the common region exceed the norm bound. However, it is easy to see that we can always choose one of these thread bundles to vanish: if $I(B: C)=0$, then we can set $v_{B \rightarrow C}=0$ (so 


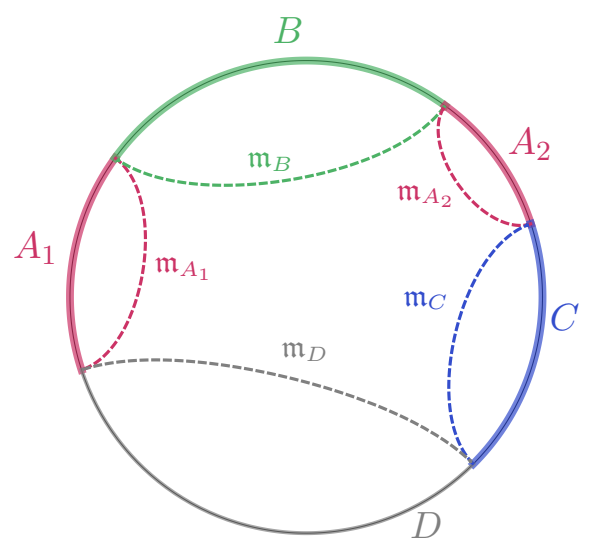

(a)

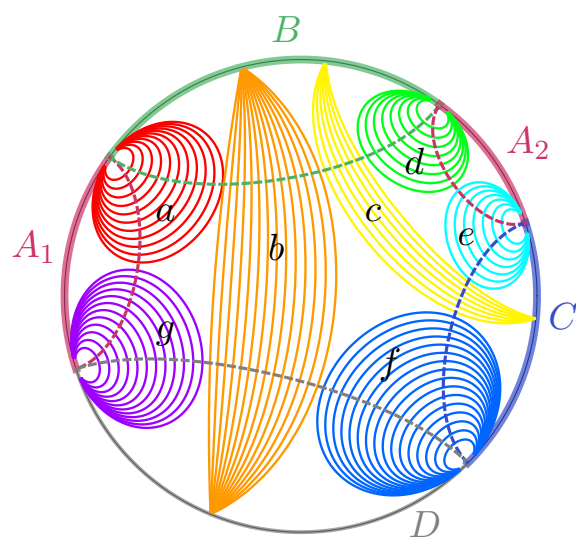

(b)

Figure 7. (a) An example of partitioning of the boundary space into 4 subsystems $A, B, C$, $D$, analogously to figure 1 , but now consisting of 5 simple regions as labeled. (b) Corresponding thread bundle construction analogous to figure 4a, which delineates the associated bulk regions $a, b, c, d, e, f, g$ connecting the requisite boundary regions as specified in the text.

in this case cooperative flows exist trivially). ${ }^{34}$ On the other hand, if $I(B: C)>0$, then the complementary regions must be uncorrelated: $I\left(A_{2}: A_{1} D\right)=0$ which means that $I\left(A_{2}: D\right)=0$, so that we can set $v_{A_{2} \rightarrow D}=0$. Let us then consider this latter case.

The first thing to check is that the requisite thread bundles can fit through the bottlenecks in a disjoint fashion. We have 5 minimal surfaces $\left(\mathfrak{m}_{A_{1}}, \mathfrak{m}_{B}, \mathfrak{m}_{A_{2}}, \mathfrak{m}_{C}\right.$, and $\left.\mathfrak{m}_{D}\right)$ and 7 bulk regions $a, b, c, d, e, f, g$ containing the 7 thread bundles which form the building blocks for the flows, which we wish to prove to be mutually non-overlapping ( $a \supset v_{A_{1} \rightarrow B}$, $b \supset v_{B \rightarrow D}, c \supset v_{B \rightarrow C}, d \supset v_{B \rightarrow A_{2}}, e \supset v_{A_{2} \rightarrow C}, f \supset v_{C \rightarrow D}$, and $\left.g \supset v_{A_{1} \rightarrow D}\right)$, cf. figure $7 \mathrm{~b}$ (which shows the actual thread construction). These thread bundles must partition the minimal surfaces so as to satisfy a set of bottleneck equations analogous to (3.6). These consist of 5 equations (one for each interval $X$ ) of the form

$$
\text { Area }\left[\mathfrak{m}_{X}\right]=\sum_{z} \operatorname{Area}\left[\mathfrak{m}_{X}^{z}\right]
$$

where $X$ denotes one of the 5 simple boundary intervals and $z$ denotes all the thread bundles with cross $\mathfrak{m}_{X}$, and 7 equations (one for each thread bundle $\mathrm{z}$ ) of the form

$$
\text { Area }\left[\mathfrak{m}_{X}^{z}\right]=\text { Area }\left[\mathfrak{m}_{Y}^{z}\right]
$$

where $X$ and $Y$ is a pair of simple boundary intervals joined by a thread bundle $z$. Since each thread bundle gives a partition for two minimal surfaces (associated with the regions the thread bundle connects), we have total of 14 partitions. Hence we have 12 equations for 14 unknowns, which gives a 2-parameter family of solutions; we leave it as an easy

\footnotetext{
${ }^{34}$ In particular, by argument of section 3.2 , we can set $v_{B}=v_{C}$ and $v_{A}=v_{A B C}$, which gives $\tilde{v}_{1}=v_{A}$ and $\tilde{v}_{2}=\tilde{v}_{3}=v_{B}$, manifesting all as flows.
} 
exercise for the reader to write out the explicit equations and confirm that a solution must necessarily exist.

So far, we have ascertained that the 5 minimal surfaces can be partitioned so as to accommodate all the thread bundles correctly. We can now employ the same arguments as in section 3.4 to verify that by using minimal surface foliations, the remainder of the thread bundles must bend away from each other. Either of our explicit constructions is generalizable, but it's most convenient to adopt the first one, using 7 global foliations. Since the reasoning presented at the end of section 3.4.3 was local in the sense of involving only two adjoining thread bundles, we can employ exactly the same argument in the present case. The explicit thread bundle construction is given in figure 7b. As for the simple case, we can guide the bundles using only the two foliation families depicted in figure $3 \mathrm{~b}$ (for bundles $a, d, e, f, g$ ) and figure $3 \mathrm{c}$ (for bundles $b$ and $c$ ). This choice again fixes the solution to the bottleneck equations uniquely. We could also introduce more freedom by using only the separated endpoint form of the bundles, or even more generally, a hybrid of the two methods. This proves the existence of a cooperative flow for the 5-region case, where the methods of section 3.2 (utilizing vanishing mutual information) would have failed.

It should be evident by now that we can generalize our construction to arbitrarily large set of boundary intervals $\left\{A_{i}\right\},\left\{B_{j}\right\},\left\{C_{k}\right\}$, and $\left\{D_{\ell}\right\}$, with arbitrary sizes and ordering. To characterize the system of equations we have to solve, it is convenient to represent any such configuration by a graph, with nodes corresponding to the boundary regions and links to the thread bundles joining them. In order for the thread bundles to be compatible, they cannot intersect, which means that the graph must be planar. ${ }^{35}$ To see that this is always possible, consider a pair of intersecting thread bundle candidates, say connecting $X \leftrightarrow Z$ and $Y \leftrightarrow W$ (in other words, the boundary contains the intervals $X, Y, Z$, and $W$ distributed somewhere along the circle in this order, but without any restriction on which subsystems they are part of), as indicated in figure 8. For labeling convenience, we'll collect the intervening regions into intervals labeled by $P, Q, R$, and $S$, though we don't require these to be non-vanishing.

We can now generalize the previous argument to show that if $X$ is correlated with $Z$, then $Y$ cannot be correlated with $W$, and vice-versa: compare

$$
\text { Area }\left[\mathfrak{m}_{X}\right]+\text { Area }\left[\mathfrak{m}_{Z}\right] \quad \text { versus } \quad \text { Area }\left[\mathfrak{m}_{P Y Q}\right]+\text { Area }\left[\mathfrak{m}_{S W R}\right]
$$

relevant for $S(X Z)$. If the l.h.s. is smaller than the r.h.s., then $I(X: Z)=0$. On the other hand, if it's larger, then $I(P Y Q: S W R)=0$ which by monotonicity and positivity implies that $I(Y: W)=0$ (and of course if the two sides are equal, both mutual informations vanish). The consequence is that we never need to invoke the $X \leftrightarrow Z$ and $Y \leftrightarrow W$ thread bundles simultaneously. This justifies our claim that the graph is planar.

Let us now use the graph representation to count the number of equations and unknowns. Suppose the graph has a total of $n$ nodes and $\ell$ links. The number of unknowns

\footnotetext{
${ }^{35}$ While planarity is not a fundamental requirement in full generality (e.g., it can easily be violated in the higher dimensional case as discussed in section 4.3), here we use it to conveniently characterize the set of constraints.
} 


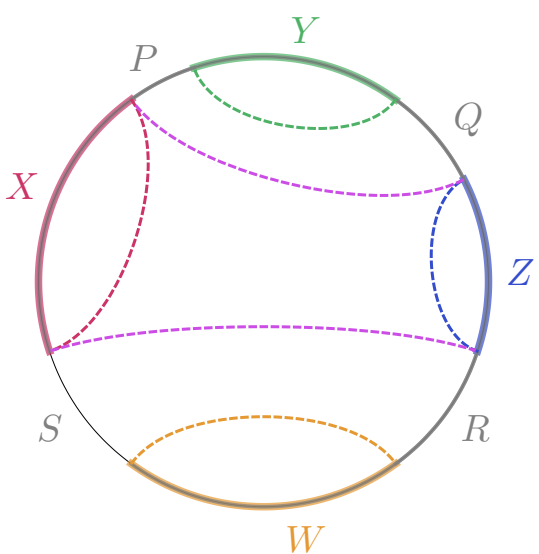

Figure 8. Generalization of figure 1 to allow our basic subsystems $A, B, C$ and $D$ to consists of arbitrarily many intervals, where we focus the potentially intersecting thread bundles for some 4 intervals $X, Y, Z$, and $W$ picked out of the basic set, separated by the remaining intervals, collected into $P, Q, R$, and $S$.

$\mathfrak{m}_{X}^{z}$ corresponds to the number of partitions of the minimal surfaces, and since each thread bundle crosses two of the minimal surfaces, this is given by $2 \ell$. The number of equations is given by $n+\ell$, one for each minimal surface and one for each thread bundle. This means that

$$
\text { (\# unknowns) }-(\# \text { equations })=\ell-n
$$

The r.h.s. is guaranteed to be non-negative: we must have at least as many links as nodes since all the adjoining regions have non-zero (in fact diverging) mutual information. This already guarantees that we should find at least one solution. Moreover, although nearlyequal size intervals could all have vanishing mutual information for any non-adjoining pair, more typically (if some regions are sufficiently large or sufficiently small), there will be additional correlations, making $\ell>n$, which would yield a $(\ell-n)$-parameter family of solutions: the more regions are correlated, the more freedom we have in choosing the thread bundles. In other words, we can shift threads between regions while maintaining the cooperative nature of the flows. (On the other hand, we don't have arbitrary freedom, since planarity of the graph also puts an upper bound on the dimensionality of the space of solutions: $\ell \leq 3 n-6$.)

As before, once we have solved the minimal surface partitioning problem, the cooperativeness of the remainder of the thread bundles follows by the local arguments of section 3.4.3. Hence for any partitioning of the boundary, with arbitrarily many regions, of arbitrary sizes and ordering, we can always construct cooperative flows.

\subsection{Exciting the geometry}

So far we have been working with a constant time slice of $\mathrm{AdS}_{3}$ geometry, corresponding to the near-vacuum (or a slightly-excited state belonging to the same 'code subspace' describing the same bulk geometry) state of the CFT, but in fact our arguments depended on this choice only in a very weak sense. In particular, we only relied on $S(A B C D)=0$ 
and the minimal surface (i.e. geodesic) foliations of the bulk. Clearly, as long as it remains true that to every boundary interval we can associate a unique bulk geodesic, that the set of these geodesics covers the entire spatial slice, and that they all end on a single boundary, our arguments will go through without modification. Moreover, even in the more general spacetime where the above conditions fail, our arguments can still be suitably accommodated.

To see the unmodified part, note that minimal surfaces anchored on boundaries of nested regions cannot intersect. ${ }^{36}$ This follows by similar arguments as used in section 3.1: if they did, then we could decrease their area further by smoothing out the corner at the intersection, thereby contradicting the assumption of minimality. We will refer to this property as nesting. Such a minimal-surface foliation then generates a maximally collimated flow, just as before. Multiple foliations (or a local foliation) allows us to construct multiple thread bundles, whose compatibility is ensured by satisfying the requisite bottleneck equations (namely (3.6) or their obvious generalization discussed in section 4.1), since the argument for the non-intersection of the distinct thread bundles follows from nesting as before.

Let us now consider sufficiently large deformations away from the $\mathrm{AdS}_{3}$ geometry (but still keeping the metric time-reflection symmetric around the time of interest), such that minimal surfaces anchored on the boundary no longer foliate the bulk. Although this can happen in a topologically and causally trivial way ${ }^{37}$ as sketched in figure $9 \mathrm{a}$, the most interesting cases which have been well-studied involve black hole geometries. As argued in e.g. [20], minimal surfaces cannot penetrate the horizon of a static black hole, and minimality along with the homology constraint leads to a phase transition in the nature of the minimal surfaces. In the so-called plateaux regime [15], surfaces anchored on large enough region $X$ consist of two disconnected components, one corresponding to a minimal surface for the complementary region (on the same boundary) $\bar{X}$, and the other wrapping the black hole horizon bifurcation surface, as indicated in figure $9 \mathrm{~b}$.

First consider the simpler case, indicated in figure 9a. This family of minimal surfaces guides a thread bundle between the left side $\varphi=\pi$ and the right side $\varphi=0$. Outside the central gap, this bundle behaves as in the previous discussion. Inside the gap, the bundle actually has more freedom. Imagine any spatial co-dimension one (in our case 1-d) 'surface', anchored on the critical entangling surface at which the phase transition occurs (in figure 9a straddling the top and bottom, $\varphi= \pm \pi / 2$, e.g. a vertical line). Any such surface has by construction larger area than the minimal surfaces at the edge of the

\footnotetext{
${ }^{36}$ In more complicated situations considered below, we could have multiple components of a given minimal surface, say including a co-dimension-2 compact part, wrapping a horizon of a black hole. In such cases, in the so-called plateaux regime [15], these compact components associated with nested boundary regions, do coincide. In higher dimensions, we could also contrive minimal surfaces to be tangent on some higher $(>2)$-codimensional locus. However, neither of these is a problem for our construction.

${ }^{37}$ For example for scalar solitons, see e.g. [16, 17]. (If the gap remains for all families of minimal surfaces, such as would be the case for spherically symmetric configurations of the above type, such regions would constitute what was dubbed 'entanglement shadows' in [18]. On the other hand, the temporal version of this effect is somewhat harder to achieve; under sufficiently strong assumptions, maximal-volume co-dimension one slices of the bulk, when anchored on boundary foliation, would provide a bulk foliation; cf. e.g. [19].)
} 


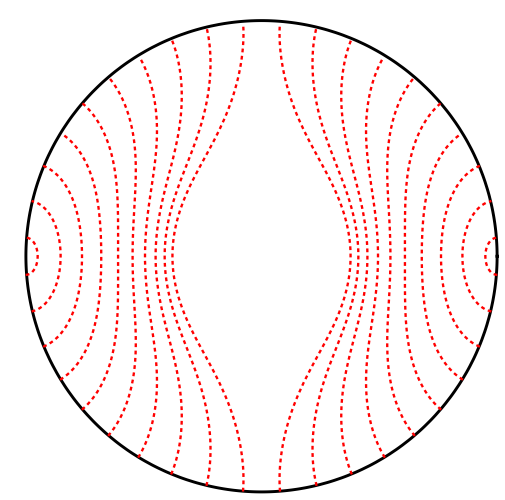

(a)

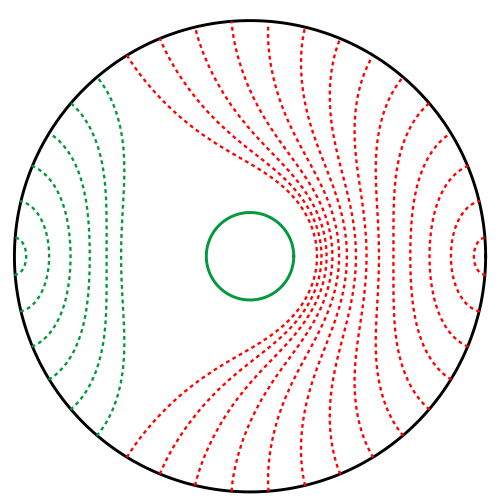

(b)

Figure 9. Examples of bulk geometries wherein a boundary foliation does not generate a bulk foliation, in the sense that minimal surfaces leave behind 'gaps'. (a) A hypothetical topologically and causally trivial 'compact star' geometry. (a) A black hole (BTZ) geometry, with horizon indicated by the thick green curve in the center. In both cases the dotted curves are globally minimal surfaces. However it the black hole case, in order to satisfy the homology requirement, the minimal surfaces for a large enough region $\left(-\varphi_{0}, \varphi_{0}\right)$ consist of two disconnected components, the dotted green curve and the horizon.

gap, and therefore cannot impose a more stringent bottleneck for the flow. We can foliate the gap by non-minimal surfaces, in which case the threads would simply spread out and refocus on the other side of the gap, or we could foliate parts of the gap by local foliations which would keep the bundle maximally collimated but possibly split into multiple strands. Employing the latter method, we can recycle the constructions explained in section 3.4.4. Hence in this class of cases we can obtain a cooperative flow.

Finally, in the topologically non-trivial case having horizons (cf. figure 9b) and multiple boundaries, the main new ingredient is that not all the treads need to end on the same AdS boundary - some can penetrate the horizon ${ }^{38}$, and emerge on another boundary. However, in such a case, we can redefine $D$ to include the entire spatial slice of the other boundary (as presaged in footnote 14), and proceed as in section 4.1 and the above arguments for not 'minding the gap'.

The upshot is that we can still guide a cooperative flow through any static $2+1$ dimensional asymptotically AdS bulk geometry, even when the minimal surface foliations leave behind gaps and the spacetime contains wormholes and boundaries (possibly ending up with more bulk regions which don't contain any bundles). To generalize further, we should consider what happens in higher dimensions, as well as what happens when we allow for general time dependence, which we address in the next two subsections.

\footnotetext{
${ }^{38}$ There is in fact a nice story regarding the bit thread version of plateaux: there are three distinct regimes characterized by the size $2 \varphi_{0}$ of the boundary region. When the region is less than half the boundary $\varphi_{0} \leq \pi / 2$, the threads need not enter the black hole at all. When it is larger than half but smaller than the critical size for the plateaux regime $\pi / 2<\varphi_{0}<\varphi_{\text {crit }}$, some threads must enter the black hole, but not necessarily saturate the norm bound there. On the other hand, in the plateaux regime $\varphi_{0} \geq \varphi_{\text {crit }}$, the threads entering the horizon are necessarily maximally collimated.
} 


\subsection{Higher dimensions}

One crucial difference between the $2+1$ dimensional spacetime we have hitherto considered and a higher-dimensional one is that while the former disallows simultaneous $A C$ and $B D$ flows on a constant-time slice (since the threads would then necessarily intersect), in the latter case both are allowed since they can go around each other in the extra direction. Hence in higher dimensions we do not have to restrict to a subset of potential thread bundles, and relatedly, we have no natural ordering of the boundary regions. If this were the only effect of higher dimensions, it would be clear that the feasibility of finding compatible flows necessarily improves with increasing dimension. However, there is another consequence of higher dimensions, which could potentially have the opposite effect: one might worry that the same bulk region has to be shared by many more boundary elements. We should therefore proceed more carefully.

We can nevertheless utilize the same key feature as in the 3-dimensional case, namely that minimal surface foliations still comb the thread bundles in a maximally collimated fashion. Recall that minimal surfaces satisfy the nesting property in any number of dimensions (indeed, we have been using the higher-dimensional language in order to make this generalization of our arguments more apparent). There will now generally be gaps in the foliation as surfaces undergo phase transitions, ${ }^{39}$ however these can be dealt with in the same way as in section 4.2. Moreover, we can also more readily utilize the large volume near the boundary to lead most of the threads through this region in a more trivial fashion.

More specifically, the bottleneck equations as such are not sensitive to dimensionality and their structure changes favorably (in the sense that each new thread bundle introduces 2 extra unknowns but only one extra equation). Using nesting, we can then see that once the bundles satisfy the compatibility conditions at the bottlenecks, the local foliations can be chosen so as to guide them away from each other in the rest of the spacetime. These considerations suggest that it is indeed easier to construct cooperative flows in higher spatial dimensions.

One curious feature of changing spacetime dimensionality which affects threads is that while in two-dimensional space threads cannot swap endpoints (because that would require them to intersect), in three dimensional space they can swap endpoints in a topologically interesting way (i.e. they can be braided), and in higher dimensions the swapping is entirely trivial. Since the RT prescription does not manifest this distinction, it would be interesting to see whether this has any actual physical implications.

\subsection{Time dependence}

So far, we have been considering static ${ }^{40}$ configurations which allowed us to compute the entanglement entropy via the Ryu-Takayanagi prescription [7] in terms of minimal surfaces $\mathfrak{m}_{X}$, or equivalently the Freedman-Headrick prescription [12] in terms of bit threads $v_{X}$.

\footnotetext{
${ }^{39}$ In fact, minimal surfaces can have various non-trivial features (for example even for simply-connected boundary regions the bulk minimal surface can have handles) and distinct surfaces may be tangent along higher co-dimension subsets.

${ }^{40}$ One can generalize this class slightly to include bulk spacetimes with time reflection symmetry, provided we restrict our consideration to the specific time slice admitting this symmetry.
} 
Even within the class of holographic theories with geometrical bulk dual, this is a severe limitation. Not only are genuinely time-dependent configurations physically relevant and interesting in their own right, but in the context of holography they might also supply a key insight to structure of the holographic mapping and thereby elucidate the emergence of bulk spacetime. This motivated generalizing of RT and FH prescriptions.

In general time-dependent situations, one can covariantize these prescriptions, which gives rise to the Hubeny-Rangamani-Takayanagi (HRT) prescription [9] in terms of extremal surfaces $\mathfrak{E}_{X}$ which can also be recast into the Wall prescription [11] in terms of 'maximin' surfaces, ${ }^{41}$ (allowing MMI to be proved in the time-dependent set-up), or the newer prescription [21] in terms of 'covariant bit threads'. The latter prescription builds on the methods of [13], using convex relaxation on the maximin prescription and Lagrangian duality to express the entropy in terms of a flux of a divergence-free flow field $v$, whose norm bound gets replaced by a more global norm bound, integrated over temporal extent. In other words, we still have 1-dimensional bit threads, which can however have a non-vanishing time component. Nevertheless, one can argue that all threads must pass through the requisite extremal surface, in a maximally-packed manner (thereby reproducing the HRT prescription). In fact, although the threads may be timelike-separated from each other elsewhere, it is likewise possible to flatten the full congruence to a single spatial surface (thereby reproducing maximin), thus allowing us to recycle the methodology developed for the static case.

Let us now apply the covariant bit thread prescription to the situation at hand. As a first step, we wish to consider a requisite family of extremal surfaces which would serve as 'thread-guide' to a given thread bundle. Since any normal congruence to extremal surfaces (whether spacelike or timelike or null) has by definition vanishing expansion, once we find a family of extremal surfaces which foliate a given Cauchy slice, we can guide our thread bundle along that slice in precisely the same manner as in the previous sections. This is indeed possible thanks to the covariant version of nesting, which in the bulk gets uplifted to a property pertaining to full co-dimension-zero regions, called entanglement wedges. ${ }^{42}$ In particular, entanglement wedges for nested boundary regions are themselves nested [11, 22], so the corresponding extremal surfaces themselves must lie on a common maximin slice. A foliating family of boundary regions then generates a Cauchy slice locally foliated by the corresponding extremal surfaces. ${ }^{43}$

This suggests that one should likewise be able to repeat the above constructions for arbitrary set of boundary regions $\{X\}$ and thread bundles $\{z\}$, by localizing the threads

\footnotetext{
${ }^{41}$ More specifically, the Maximin prescription of [11] retains some useful features of RT, while restoring covariance in two steps: first, on any bulk Cauchy slice $\Sigma$ whose boundary contains the entangling surface, we consider the area of a globally minimal surface homologous to the boundary region. Second the entanglement entropy is given by maximizing this area over all Cauchy surfaces. The resulting maximin surface realizing this maximum (under certain physically reasonable assumptions) is the HRT extremal surface, and any Cauchy slice within which this extremal surface minimizes the area is called a maximin slice.

${ }^{42}$ Entanglement wedge (first introduced in [22]) for a bulk region $X$ is the domain of dependence of the bulk homology region $r_{X}$ (part of a bulk Cauchy slice such that $\partial r_{X}=X-\mathfrak{m}_{X}$ ), or equivalently the set of spacelike-separated bulk points between $X$ and $\mathfrak{m}_{X}$.

${ }^{43}$ More generally the extremal surface family could leave gaps along the slice, but we can apply the same reasoning as in section 4.2 to this case.
} 
to specific spatial slices of the bulk. In particular, consider spacelike (maximin) slices $\Sigma_{z}$, foliated by extremal surfaces (anchored on a nested set of boundary regions whose entangling surfaces in turn foliate the boundary), which comb the bit threads in a maximally collimated fashion. While different boundary-foliating families of entangling surfaces will generally give rise to different bulk Cauchy slices (which can then be generically timelike separated from each other), by construction these slices must intersect at the common extremal surfaces $\mathfrak{E}_{X}$. This means that all the thread bundles constructed from such foliations will encounter the joint bottlenecks at the $\left\{\mathfrak{m}_{X}\right\}$. To check for the consistency of the cooperative flow at the bottlenecks, we use the same equations (analogous to (3.6)) for the partitions of the extremal surfaces by $\mathfrak{m}_{X}^{z}$, and hence by the same arguments a solution to this set of equations exists. Moreover, entanglement wedge nesting also guarantees that two adjoining thread bundles $z$ and $z^{\prime}$ passing normally through $\mathfrak{m}_{X}$ will be 'steered away' from each other by the respective foliations as long as the boundary regions behave as prescribed for the static case.

Thus we can apparently uplift the arguments and constructions of cooperative flows from the static case to a generic time-dependent case, thereby vastly enlarging its regime of validity to states for which HRT applies. However, it would be interesting to consider whether we can obtain more interesting behavior by not flattening the thread bundles to Cauchy slices. We leave this problem for future exploration.

\section{Discussion}

Since the geometric proof of SSA and MMI using the HRT prescription does not manifest the profound difference between these two inequalities while the bit thread formulation of [12] seems to distinguish them more strikingly, we have focused on proving MMI (1.5) using bit threads. In particular, we demonstrated the existence of disjoint thread bundles which constitute the building blocks of cooperative flows, which in turn guarantee that MMI holds. Although we detailed the construction explicitly for $\mathrm{AdS}_{3}$ geometry, we conjectured that our method of obtaining cooperative flows applies to any bulk geometry for which HRT applies, and indicated how each type of generalization should work. If this conjecture holds, it proves MMI in the bit thread formulation in equal generality as achieved previously $[3,11]$ using the RT/HRT prescription.

However, the connection to the first part of the title has hitherto remained tenuous. In this section we revisit the relation to bulk locality, and speculate on some emerging lessons and open problems.

Bulk locality: our proof of MMI involved constructing maximally collimated disjoint thread bundles. To this end, we have utilized minimal surface foliations of bulk regions, since their normal congruences have vanishing expansions. The fact that minimal surfaces anchored on nested regions cannot intersect has ensured that these thread bundles are well-defined everywhere. However, here this nesting property has been applied more locally than previously. For example the bit thread proof of SSA [12] used nesting in the sense that there exists a common maximizer flow for nested regions. In [13], this was shown 
to be equivalent to the statement that bulk 'homology regions' $\left\{r_{X}\right\}$ corresponding to nested boundary regions $\{X\}$ are themselves nested (or equivalently the associated minimal surfaces $\left\{\mathfrak{m}_{X}\right\}$ don't intersect). In both formulations, however, the starting assumption of nesting pertained to the boundary. As explained in [12], this form of nesting does not suffice to prove MMI. In retrospect, the reason for this seems to lie in that it is too global. We needed an additional ingredient, namely further details of the bulk geometry. In this sense, the fact that MMI holds for CFT states with geometric dual, and not necessarily in greater generality, naturally stems from the essence of the geometry: classically, it is local.

We however emphasize that MMI as such does not imply bulk locality, as has already been appreciated in [3]; in fact even for 4-qubit systems (which one might think are maximally removed from anything resembling holography), MMI is generically upheld [23]. On the other hand, in the holographic context, it is interesting to ask what effect would stringy and quantum corrections have on our construction. From arguments based on RT [3], one expects that MMI holds whenever the bulk is classical, regardless of the actual field equations governing the geometry at leading order in $N^{2}$, but not once the quantum effects become important. ${ }^{44}$ These expectations are consistent with the bit thread picture. As shown in [25], the effect of adding higher-curvature terms to the gravitational action manifests itself in corrections to the norm bound: the bit thread thickness effectively changes in response to the local geometry and thread orientation. However, since minimization (in the static case) is involved in determining the bottlenecks, one still retains a type of nesting, so one should still be able to guide the thread bundles through the bulk in a cooperative fashion. On the other hand, finite- $N$ effects modify the divergencelessness condition [12], so threads can disappear and reappear at various points in the spacetime. This invalidates our construction based on unbroken threads entirely, though a-priori the sign of this effect is unclear: one would expect a larger set of possibilities for a quantum flow.

Role of monogamy: a key feature of quantum entanglement is the monogamy property: maximum entanglement between two parties cannot be shared by a third party. As mentioned in the Introduction, this property would guarantee the negativity of tripartite information $I_{3}$ (and hence MMI) if the mutual information only consisted of quantum entanglement. Let us see if we can turn this intuition around, to further explore the implications of MMI being upheld in holography. In particular, we would like to ask if there is some sense in which the bulk can be pictured as arising from purely monogamous entanglement. ${ }^{45}$

Note that the bit threads, having only two ends and no junctions, are rather evocative of monogamy: their endpoints cannot be shared by a third party just as quantum entan-

\footnotetext{
${ }^{44}$ Even the subleading $1 / N$ corrections in terms of bulk entanglement between the homology regions [24] a-priori need not uphold MMI.

${ }^{45}$ Indeed, phrased at this abstract level, this speculation is not dissimilar from previous statements in the literature such as spacetime being built up from quantum entanglement [26] or ER=EPR [27]. (We observe in passing that this is a-priori rather different from the ideas of the type discussed in e.g. [28] involving kinematic space where bulk geodesics played a crucial role. We emphasize that our thread bundles are generally not composed of geodesics, since by geodesic deviation equation the latter wouldn't remain equidistant.)
} 
glement cannot be shared. Could the threads then 'weave the fabric of spacetime' in some concrete sense? This is in fact reminiscent of some of the expectations regarding tensor networks in holography [29], though one would want this to operate at a much more local level. An immediate objection to this naive picture is that the flows depend on not just the state of the full system but also on the specified partition, whereas the bulk geometry is independent of the latter. An associated puzzle is that if we interpret the threads as implementing a purely bipartite (as opposed to multipartite) structure of entanglement, one would conclude that the tripartite information then necessarily vanishes, i.e. MMI would necessarily be saturated, contradicting explicit computations.

Let us examine this issue a bit further. We have seen that although maximizer flows for correlated (disjoint) regions are incompatible, the thread bundles which these are built out of can nevertheless cooperate. If we could play the same trick not only with $v_{A}, v_{B}, v_{C}$, and $v_{A B C}$ as in our construction, but also simultaneously with $v_{A B}, v_{B C}$, and $v_{A C}$, then all the bundles appearing in MMI (1.5) would explicitly cancel. We however manifestly cannot decompose flows into thread bundles in such a way as to reassemble all of these maximizer flows from the same set of bundles, simply because the associated minimal surfaces, which the bundles would have to traverse perpendicularly and with unit norm, intersect each other. Interestingly, using the method of section 3.4.4 we could construct a cooperative flow out of thread bundles which pass through each of the evoked extremal surfaces, including both $\mathfrak{m}_{A B}$ and $\mathfrak{m}_{B C}$, perpendicularly. ${ }^{46}$ However, this does not suffice for such bundles to be maximizer flows for all of the associated regions, because the above construction would leave part of $\mathfrak{m}_{A B}$ and $\mathfrak{m}_{B C}$ (at the least in the vicinity of their intersection) untraversed. This explains how bit threads can indeed implement monogamy naturally while at the same time being compatible with non-trivial multipartite entanglement structure.

Other entanglement relations and constructs: the above observations suggest that bulk locality should suffice to prove entanglement relations which are guaranteed to hold by monogamy (in the sense of mutual information being dominated by quantum entanglement as opposed to classical correlations). One such interesting relation is the 5-party cyclic inequality (which can be thought of as a generalization of MMI) [2]. Since this inequality has hitherto likewise eluded proof using bit threads, in appendix A we offer a few comments on generalizing our methods to that case. The argument of the inequality being guaranteed by existence of cooperative flows is in fact directly analogous to that of section 2. However, the actual construction of requisite cooperative flows has to be modified from that discussed above, due to the novel feature of evoking simultaneous maximizer flows for overlapping regions, which (by essentially the same arguments as in the previous paragraph) cannot

\footnotetext{
${ }^{46}$ For example, if we want to construct cooperative flow which passes $\mathfrak{m}_{A B}$ perpendicularly, we merely need to extend the orange fanned-out foliations in figure 6 into the central region, so that they both contain $\mathfrak{m}_{A B}$. More specifically, we extend the foliation in the $(\partial A \cap \partial D)-p_{b e}-p_{a b e}-p_{a e}$ quadrilateral region into the lower portion of the central hexagonal region (below $\mathfrak{m}_{A B}$ ), and similarly extend the $(\partial B \cap \partial C)-p_{b c}-p_{b c d}-p_{c d}$ quadrilateral region into the upper portion of the central hexagonal region (above $\mathfrak{m}_{A B}$ ). By a similar trick (with additional foliations fanning out from the central point), one could even guide our thread bundles perpendicularly through both $\mathfrak{m}_{A B}$ and $\mathfrak{m}_{B C}$, effectively splitting the $v_{B \rightarrow D}$ bundle to bypass the disallowed neighborhood of their intersection.
} 
be achieved with a single set of disjoints bundles. Though it appears possible to achieve with multiple sets of strands, we leave the explicit construction for future work. It will be interesting to elucidate the additional property of bulk locality (if sufficient) to manifest cooperative flows in this case.

One might in fact hope that the full set of such higher-partite entanglement relations (defining the holographic entropy cone [2]) would contain sufficient set of specifications of the requisite entanglement structure to identify geometric states directly, which partly motivates the search for further such relations. ${ }^{47}$ It would be interesting to see if, for all relations following from the existence of cooperative flows, the requirement of realizability of these via maximally collimated disjoint thread bundles, in fact aids in this quest.

Let us close with a more general comment. Originally the bit thread prescription of [12] was formulated with the explicit goal to provide an alternate prescription for entanglement entropy. In the present work we have utilized bit threads accordingly, since we have only considered quantum information theoretic constructs built out of entanglement entropy (most generally these comprise the information quantities mentioned above in the context of the holographic entropy cone). However, there are many other interesting and useful information theoretic quantities which are not directly constructed from entanglement entropy. As such, a-priori, the bit threads need not have any useful relation to such quantities. However, we find the above constructions of thread bundles highly encouraging for finding wider applications of bit threads. In particular, our constructions are rather reminiscent of the holographic entanglement of purification proposed in [31]. Furthermore, since bit threads might also be cognizant of certain aspects of the quantum / classical separation, it would be interesting to explore whether they bear any connection to concepts such as relative entropy of entanglement, quantum discord, robustness, entanglement of distillation, etc.. These in turn might reveal useful insights into the emergence of bulk spacetime in holography.

\section{Acknowledgments}

It is a great pleasure to thank Matt Headrick, Juan Maldacena, Mukund Rangamani, Max Rota, and Tadashi Takayanagi for stimulating discussions. I would also like to thank Matt Headrick for comments on an earlier version of the draft. I am grateful to the Kavli Institute for Theoretical Physics in Santa Barbara, the Centro Atomico Bariloche, ICTSTIFR in Bengaluru, the Galileo Galilei Institute in Florence, and the Yukawa Institute for Theoretical Physics at Kyoto University, for hospitality during various stages of this project. This work was supported by U.S. Department of Energy grant DE-SC0009999 and by funds from the University of California.

\footnotetext{
${ }^{47}$ These are known exhaustively for only up to 5 parties, and generalizations of some of the known relations (such as the 5-party cyclic inequality) to arbitrarily large (but in the cyclic case odd) number of parties already guarantee infinitely many independent inequalities [2]. (The upcoming work initiated by [30] develops a program to generate further such relations and corresponding information quantities efficiently.)
} 


\section{A 5-party cyclic inequality}

Hitherto we have focused attention on MMI, which is an entropy inequality that involves partitioning the Hilbert space into 4 parts, which we denoted $A, B, C$, and $D \equiv \overline{A B C}$. However, our geometric constructs as such didn't rely on this number of partitions, so one would expect the methodology to be applicable more universally. As an illustrative example, consider the 5-region cyclic inequality, which can be thought of as a generalization of MMI [2]. For 6 partitions, $A, B, C, D, E$, and the purifier $F \equiv \overline{A B C D E}$, the cyclic inequality states

$$
\begin{array}{r}
S(A B C)+S(B C D)+S(C D E)+S(D E A)+S(E A B) \geq \\
S(A B)+S(B C)+S(C D)+S(D E)+S(E A)+S(A B C D E)
\end{array}
$$

Performing a similar exercise as in (2.5), we can bound the l.h.s. of (A.1) by

$$
\begin{aligned}
& \int_{A B C} v_{A B C}+\int_{B C D} v_{B C D}+\int_{C D E} v_{C D E}+\int_{D E A} v_{D E A}+\int_{E A B} v_{E A B} \\
\geq & \int_{A B C} v_{1}+\int_{B C D} v_{2}+\int_{C D E} v_{3}+\int_{D E A} v_{4}+\int_{E A B} v_{5} \\
= & \int_{A}\left(v_{1}+v_{4}+v_{5}\right)+\int_{B}\left(v_{1}+v_{2}+v_{5}\right)+\int_{C}\left(v_{1}+v_{2}+v_{3}\right)+\int_{D}\left(v_{2}+v_{3}+v_{4}\right)+\int_{E}\left(v_{3}+v_{4}+v_{5}\right)
\end{aligned}
$$

where the inequality holds for any flows $v_{1}, v_{2}, v_{3}, v_{4}, v_{5}$. We want to make the last line of (A.2) to be equal to the r.h.s. of (A.1), i.e.

$$
\begin{aligned}
& \int_{A B} v_{A B}+\int_{B C} v_{B C}+\int_{C D} v_{C D}+\int_{D E} v_{D E}+\int_{E A} v_{E A}+\int_{A B C D E} v_{A B C D E}= \\
= & \int_{A}\left(v_{A B}+v_{E A}+v_{A B C D E}\right)+\int_{B}\left(v_{A B}+v_{B C}+v_{A B C D E}\right)+ \\
& +\int_{C}\left(v_{B C}+v_{C D}+v_{A B C D E}\right)+\int_{D}\left(v_{C D}+v_{D E}+v_{A B C D E}\right)+ \\
& +\int_{E}\left(v_{D E}+v_{E A}+v_{A B C D E}\right)
\end{aligned}
$$

which we can achieve if we can define

$$
\begin{aligned}
& v_{1}=\frac{1}{3}\left(v_{A B}+v_{B C}+v_{C D}-2 v_{D E}+v_{E A}+v_{A B C D E}\right) \\
& v_{2}=\frac{1}{3}\left(v_{A B}+v_{B C}+v_{C D}+v_{D E}-2 v_{E A}+v_{A B C D E}\right) \\
& v_{3}=\frac{1}{3}\left(-2 v_{A B}+v_{B C}+v_{C D}+v_{D E}+v_{E A}+v_{A B C D E}\right) \\
& v_{4}=\frac{1}{3}\left(v_{A B}-2 v_{B C}+v_{C D}+v_{D E}+v_{E A}+v_{A B C D E}\right) \\
& v_{5}=\frac{1}{3}\left(v_{A B}+v_{B C}-2 v_{C D}+v_{D E}+v_{E A}+v_{A B C D E}\right)
\end{aligned}
$$

such that all the $v_{i}$ 's on the l.h.s. of (A.4) are flows. 


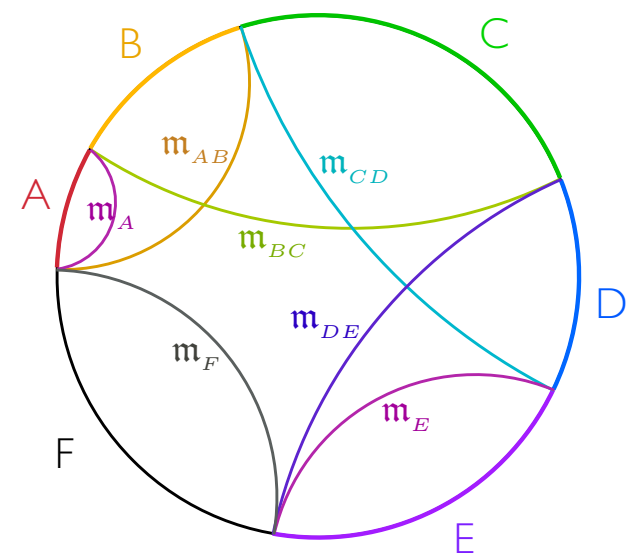

Figure 10. Analogous to figure 1: an example of partitioning of the boundary space into six regions $A, B, C, D, E$, and the complement $F \equiv \overline{A B C D E}$, as labeled. The corresponding minimal surfaces for $A B, B C, C D, D E$, and $E A$ are also indicated. The $A C$ minimal surface assumes (A.5).

Let us consider a simple example of this scenario, analogous to section 3 , with $A, B$, $C, D$, and $E$ being adjoining intervals on $\mathrm{AdS}_{3}$ boundary, as indicated in figure 10, which also shows the minimal surfaces for $A B, B C, C D, D E$, and $E A$. Note that to draw the relevant minimal surface for $A E$ as $\mathfrak{m}_{A} \cup \mathfrak{m}_{E}$, we assumed that

$$
\text { Area }\left[\mathfrak{m}_{B C D}\right]+\text { Area }\left[\mathfrak{m}_{F}\right] \geq \text { Area }\left[\mathfrak{m}_{A}\right]+\text { Area }\left[\mathfrak{m}_{E}\right] \text {. }
$$

We immediately observe a novel aspect compared to the MMI case: unlike the previous situation (cf. figure 2) where we had a single set of thread bundles which we could use for all the $v_{i}$ 's, we now have expressions involving flows through intersecting minimal surfaces. This means that a single set of thread bundles does not suffice, i.e. we cannot have a fixed set of bit threads which would maximize the flow through $A B$ and through $B C$ simultaneously since $\mathfrak{m}_{A B}$ intersects $\mathfrak{m}_{B C}$ with different normal direction.

Nevertheless, if we evaluate the five expressions (A.4) on the six bottlenecks, they appear to be mutually compatible. This suggests that a cooperative flow construction should be possible, albeit utilizing a more complicated method, evoking multiple superimposed thread bundles. We however we leave an explicit construction for future work.

Open Access. This article is distributed under the terms of the Creative Commons Attribution License (CC-BY 4.0), which permits any use, distribution and reproduction in any medium, provided the original author(s) and source are credited.

\section{References}

[1] M. Headrick, General properties of holographic entanglement entropy, JHEP 03 (2014) 085 [arXiv: 1312.6717] [INSPIRE].

[2] N. Bao, S. Nezami, H. Ooguri, B. Stoica, J. Sully and M. Walter, The Holographic Entropy Cone, JHEP 09 (2015) 130 [arXiv:1505.07839] [INSPIRE]. 
[3] P. Hayden, M. Headrick and A. Maloney, Holographic Mutual Information is Monogamous, Phys. Rev. D 87 (2013) 046003 [arXiv: 1107.2940] [InSPIRE].

[4] E. Witten, A Mini-Introduction To Information Theory, arXiv:1805.11965 [INSPIRE].

[5] T. Takayanagi, T. Ugajin and K. Umemoto, Towards an Entanglement Measure for Mixed States in CFTs Based on Relative Entropy, JHEP 10 (2018) 166 [arXiv:1807.09448] [INSPIRE].

[6] S. Ryu and T. Takayanagi, Aspects of Holographic Entanglement Entropy, JHEP 08 (2006) 045 [hep-th/0605073] [INSPIRE].

[7] S. Ryu and T. Takayanagi, Holographic derivation of entanglement entropy from AdS/CFT, Phys. Rev. Lett. 96 (2006) 181602 [hep-th/0603001] [INSPIRE].

[8] E. Witten, APS Medal for Exceptional Achievement in Research: Invited article on entanglement properties of quantum field theory, Rev. Mod. Phys. 90 (2018) 045003 [arXiv: 1803.04993] [INSPIRE].

[9] V.E. Hubeny, M. Rangamani and T. Takayanagi, A Covariant holographic entanglement entropy proposal, JHEP 07 (2007) 062 [arXiv:0705.0016] [INSPIRE].

[10] M. Headrick and T. Takayanagi, A Holographic proof of the strong subadditivity of entanglement entropy, Phys. Rev. D 76 (2007) 106013 [arXiv:0704.3719] [INSPIRE].

[11] A.C. Wall, Maximin Surfaces and the Strong Subadditivity of the Covariant Holographic Entanglement Entropy, Class. Quant. Grav. 31 (2014) 225007 [arXiv:1211.3494] [InSPIRE].

[12] M. Freedman and M. Headrick, Bit threads and holographic entanglement, Commun. Math. Phys. 352 (2017) 407 [arXiv: 1604.00354] [INSPIRE].

[13] M. Headrick and V.E. Hubeny, Riemannian and Lorentzian flow-cut theorems, Class. Quant. Grav. 35 (2018) 10 [arXiv:1710.09516] [INSPIRE].

[14] S.X. Cui, P. Hayden, T. He, M. Headrick, B. Stoica and M. Walter, Bit Threads and Holographic Monogamy, arXiv:1808.05234 [INSPIRE].

[15] V.E. Hubeny, H. Maxfield, M. Rangamani and E. Tonni, Holographic entanglement plateaux, JHEP 08 (2013) 092 [arXiv: 1306.4004] [INSPIRE].

[16] F. Nogueira, Extremal Surfaces in Asymptotically AdS Charged Boson Stars Backgrounds, Phys. Rev. D 87 (2013) 106006 [arXiv:1301.4316] [InSPIRE].

[17] S.A. Gentle and M. Rangamani, Holographic entanglement and causal information in coherent states, JHEP 01 (2014) 120 [arXiv:1311.0015] [INSPIRE].

[18] V. Balasubramanian, B.D. Chowdhury, B. Czech and J. de Boer, Entwinement and the emergence of spacetime, JHEP 01 (2015) 048 [arXiv: 1406.5859] [INSPIRE].

[19] J. Couch, S. Eccles, T. Jacobson and P. Nguyen, Holographic Complexity and Volume, JHEP 11 (2018) 044 [arXiv: 1807. 02186] [InSPIRE].

[20] V.E. Hubeny, Extremal surfaces as bulk probes in AdS/CFT, JHEP 07 (2012) 093 [arXiv: 1203.1044] [INSPIRE].

[21] M. Headrick and V.E. Hubeny, Covariant bit threads, to appear.

[22] M. Headrick, V.E. Hubeny, A. Lawrence and M. Rangamani, Causality Ėamp; holographic entanglement entropy, JHEP 12 (2014) 162 [arXiv:1408.6300] [INSPIRE]. 
[23] M. Rangamani and M. Rota, Entanglement structures in qubit systems, J. Phys. A 48 (2015) 385301 [arXiv: 1505.03696] [INSPIRE].

[24] T. Faulkner, A. Lewkowycz and J. Maldacena, Quantum corrections to holographic entanglement entropy, JHEP 11 (2013) 074 [arXiv:1307.2892] [INSPIRE].

[25] J. Harper, M. Headrick and A. Rolph, Bit Threads in Higher Curvature Gravity, arXiv: 1807.04294 [INSPIRE].

[26] M. Van Raamsdonk, Building up spacetime with quantum entanglement, Gen. Rel. Grav. 42 (2010) 2323 [arXiv: 1005.3035] [INSPIRE].

[27] J. Maldacena and L. Susskind, Cool horizons for entangled black holes, Fortsch. Phys. 61 (2013) 781 [arXiv:1306.0533] [INSPIRE].

[28] B. Czech, L. Lamprou, S. McCandlish and J. Sully, Integral Geometry and Holography, JHEP 10 (2015) 175 [arXiv:1505.05515] [INSPIRE].

[29] B. Swingle, Entanglement Renormalization and Holography, Phys. Rev. D 86 (2012) 065007 [arXiv: 0905.1317] [INSPIRE].

[30] V.E. Hubeny, M. Rangamani and M. Rota, Holographic entropy relations, Fortsch. Phys. 66 (2018) 1800067 [arXiv: 1808.07871] [InSPIRE].

[31] K. Umemoto and T. Takayanagi, Entanglement of purification through holographic duality, Nature Phys. 14 (2018) 573 [arXiv:1708.09393] [INSPIRE]. 